\title{
Well balanced schemes versus fractional step method for hyperbolic systems with source terms
}

\author{
Thierry Gallouet ${ }^{2}$, Jean-Marc Hérard ${ }^{1,2, *}$, Olivier Hurisse ${ }^{1,2}$, Alain-Yves LeRoux ${ }^{3}$ \\ ${ }^{1}$ Électricité de France, Division Recherche et Développement, \\ Département Mécanique des Fluides, Energies et Environnement, \\ 6 quai Watier, 78401 Chatou cedex, FRANCE \\ Jean-Marc.Herard@edf.fr, Olivier.Hurisse@edf.fr \\ Tel: (+33) 1-30 $877037,(+33)$ 1-30 877043 \\ ${ }^{2}$ Université de Provence, Centre de Mathématiques et d'Informatique, \\ Laboratoire d'Analyse, Topologie et Probabilités - UMR CNRS 6632-, \\ 39 rue Joliot Curie, 13453 Marseille cedex 13, FRANCE \\ gallouet@cmi.univ-mrs.fr \\ Tel: (+33) 4-91 113550 \\ ${ }^{3}$ Université de Bordeaux I, LABAG -UMR CNRS 5467-, \\ Institut de Mathématiques -FED CNRS 2254-, \\ 351, Cours de la Libération, 33405 Talence cedex , FRANCE \\ alain-yves.leroux@math.u-bordeaux1.fr \\ Tel: (+33) 5- 40006069
}

\begin{abstract}
The paper is devoted to the analysis of the real accuracy of different schemes when computing a simple hyperbolic model with source terms, which describes the motion of two-phase flows including source terms. The strategy of upwinding the source terms is investigated and compared with the standard fractional step method. A first scheme relies on the usual fractional step approach. A second scheme applies for upwinding of source terms. This one however does not provide satisfactory results when computing some specific unsteady cases. This behavior can be easily explained. It thus motivates to introduce a third scheme, which is similar to the previous one but aims at providing an increased accuracy on coarse meshes when computing highly unsteady flows. This latter scheme requires to define a cell scheme which computes the void fraction with help of a modified governing equation, while using the same interface solver. A detailed numerical study which includes a measure of the $L^{1}$ norm of the error completes the whole.

*: corresponding author
\end{abstract}




\section{Introduction}

A first result for the discretisation of source terms for hyperbolic conservation laws was given by Roe in 1986 [26], introducing the idea of upwinding of source terms. Afterwards, an extensive literature has been devoted to the numerical treatment of steady states with well balanced schemes, following ideas introduced by Greenberg and Leroux [17] (see [14],[15], [16], [23] too). The main objective in these well-balanced schemes is to preserve steady solutions on coarse meshes. In particular, it has been successfully used to deal with shallow water equations with topography, or similarly to handle one dimensional Euler steady computations in nozzles (see [10] and [22] for instance). It has also been checked that the well-balanced schemes enable to reach a steady state, as a limit for large time, of an unsteady solution. The basic idea is roughly the following: if source terms exactly balance convective effects, source terms have to be upwinded in accordance with upwinded convective fluxes.

Owing to this great success, it also seems appealing to use the well-balanced schemes to compute unsteady flows, and meanwhile benefit from the previous properties for large time. We show here, on the basis of particular initial conditions for a given system (which contains a relaxation time in the source term), that the unsteady approximation predicted by the well-balanced scheme may be far less accurate than the one given for instance by the standard fractional step method ([28]), although both of them converge towards the same solution, as the mesh size vanishes, and with the same rate of convergence. Actually, depending on the system and its initial conditions, there may be a balance between the time derivative and the source term, or a balance between the space derivative and the time derivative, depending on $(x, t)$.

In order to investigate the true accuracy of the fractional step method and the methods involving an upwinding of source terms when computing unsteady solutions, we will focus on a particular model. The model which will be examined herein is a three-equation model, and it issues from the two-phase flow framework. This model contains a source term which accounts for mass transfer between phases. The source term represents a relaxation term to some thermodynamical equilibrium. The associated relaxation time essentially depends whether one accounts for condensation, flashing or evaporation effects. As a consequence, the stiffness of source terms is variable, and may be dominant over convective effects, or on the contrary be completely negligible. This kind of source should not be confused with other sources such as effects of topography in shallow water equations, which do not involve external time scales.

Once the model is introduced, we will focus on three distinct schemes :

- the first scheme relies on the well-known fractional step approach, and it simply consists in two steps. In the first step, advective terms are accounted for, whereas in the second step, (possibly stiff) source terms are approximated. The main advantages are the following. The resulting scheme is much stable ; one may apply "standard" hyperbolic schemes to discretize first-order terms ; eventually, this decouples effects so that users who are not familiar with upwinding techniques may concentrate on the sources,

- the second scheme is the classical well-balanced scheme, which has been introduced some time ago in order to compute steady nonhomogeneous hyperbolic systems. Source terms are "upwinded", in order to represent steady states on coarse meshes in a very accurate way. This has been motivated by the rather poor behaviour (more precisely the poor accuracy) of 
the fractional step approach (noted $S_{F S}$ afterwards) in steady situations.

- the third scheme is a new scheme. It is based on a slight modification of the standard well-balanced scheme, and it involves a modification of the unknowns which are computed.

All cell schemes and interface solvers will be detailed. Interface solvers rely on the approximate Godunov approach ([4], [9]), but we insist that conclusions would be the same if one uses the exact Godunov scheme ([13]) instead. The measure of the $L^{1}$ norm of the error in unsteady test cases enables to get a precise idea of true advantages and drawbacks of the three schemes.

It will clearly appear that none among the two strategies may be disregarded, since:

(i) the fractional step method behaves rather well in all unsteady situations on coarse meshes,

(ii) the upwind approach should be prefered when steady approximations are searched on coarse meshes,

(iii) a slight modification of well-balanced schemes may drastically improve the accuracy of approximations of unsteady solutions on coarse meshes. 


\section{A simplified model to describe two-phase flows}

We consider a simple two-phase flow model which describes the motion of a mixture including two components with mass transfer terms. The convective part of the model is in conservative form, and we define the conservative variable $W={ }^{t}(\rho, \rho \alpha, \rho u)$, where $\rho$ stands for the mean density of the mixture, $u$ is the mean velocity in the field, and $\alpha$ is the concentration of one component :

$$
\begin{array}{r}
\frac{\partial \rho}{\partial t}+\frac{\partial \rho u}{\partial x}=0 \\
\frac{\partial \rho \alpha}{\partial t}+\frac{\partial \rho \alpha u}{\partial x}=\rho s(\alpha) \\
\frac{\partial \rho u}{\partial t}+\frac{\partial\left(\rho u^{2}+P(\rho)\right)}{\partial x}=\frac{\partial\left(\mu \frac{\partial u}{\partial x}\right)}{\partial x}
\end{array}
$$

The source term $s$ depends on $\alpha$ and involves some constant time scale $\tau_{0}, \mu$ is a positive constant, and the function $P(\rho)$ is increasing, and such that $P(0)=0$. The homogeneous flux is $F(W)=$ $\left(\rho u, \rho \alpha u, \rho u^{2}+P(\rho)\right)$.

The void fraction $\alpha(x, t)$ should lie in $[0,1]$. This will be refered to as the bound constraints in the following. In order to clarify the presentation, we will from time to time specify some form for the source term $s(\alpha)$, that is:

$$
s(\alpha)=\frac{\alpha_{e q}-\alpha}{\tau_{0}}
$$

with $\alpha_{e q}$ in $I_{a d m}=[0,1]$. We now describe the entropy inequality which is valid for regular solutions.

Property 1

$\overline{\text { An entropy }}$ pair $\left(\eta(W), F_{\eta}(W)\right)$ for the system (1)-(3) is ,

$$
\begin{array}{r}
\eta(W)=\frac{\rho u^{2}}{2}+\rho \psi_{1}(\rho)+\rho \psi_{2}(\alpha) \\
F_{\eta}(W)=(\eta(W)+P(\rho)) u
\end{array}
$$

with $\psi_{1}(\rho)=\int_{0}^{\rho}\left(\frac{P(a)}{a^{2}} d a\right)$ and $\psi_{2}(\alpha)=-\int_{0}^{\alpha} s(a) d a$. The entropy $\eta(W)$ is strictly convex with respect to $W$, if and only if $s^{\prime}(\alpha)<0$. The following entropy inequality holds for regular solutions of (1)-(3):

$$
\frac{\partial \eta(W)}{\partial t}+\frac{\partial F_{\eta}(W)}{\partial x}-\frac{\partial\left(\mu u \frac{\partial u}{\partial x}\right)}{\partial x}<-\rho s^{2}
$$

Introducing as usual the speed of sound waves $c$ as:

$$
c(\rho)=\left(P^{\prime}(\rho)\right)^{1 / 2}
$$

one may check that the homogeneous convective set (or left hand side) of system (1)-(3):

$$
\begin{aligned}
\frac{\partial \rho}{\partial t}+\frac{\partial \rho u}{\partial x} & =0 \\
\frac{\partial \rho \alpha}{\partial t}+\frac{\partial \rho \alpha u}{\partial x} & =0 \\
\frac{\partial \rho u}{\partial t}+\frac{\partial\left(\rho u^{2}+P(\rho)\right)}{\partial x} & =0
\end{aligned}
$$


is hyperbolic, since eigenvalues are real and distinct unless vacuum arises. Eigenvalues are:

$$
\begin{array}{r}
\lambda_{1}=u-c \\
\lambda_{2}=u \\
\lambda_{3}=u+c
\end{array}
$$

Fields 1 and 3 are genuinely non linear, and the 2 field is linearly degenerated. The entropy inequality ensures that the jump of $u$ is negative through shock waves: $u_{r}-u_{l}<0$, if subscripts $l, r$ respectively denote the states on the left and right side of the traveling shock wave.

Before going further on, we recall that the one dimensional Riemann problem corresponds to the initial value problem associated with the hyperbolic system:

$$
\frac{\partial W}{\partial t}+\frac{\partial F(W)}{\partial x}=0
$$

and the discontinuous initial data:

$$
W(x, t=0)=W_{L} \quad \text { if: } x<0 \quad \text { and: } \quad W(x, t=0)=W_{R} \quad \text { if: } x>0
$$

Everywhere in the following of the manuscript, the subscripts $L$ and $R$ will refer to the left and right states of the initial condition $W_{L}$ and $W_{R}$.

Within the class of self-similar functions $W(x, t)=w(x / t)$ composed of constant states separated by shock waves, rarefaction waves and contact discontinuities, the construction of the entropyconsistent solution of the one-dimensional Riemann problem associated with the set $((6),(8))$ with no vacuum occurrence (i.e. $\rho>0$ ) is classical, and thus is not recalled herein (see [27] for instance). Actually, the existence and uniqueness of this solution is ensured if and only if the initial data agrees with:

$$
u_{R}-u_{L}<g\left(\rho_{L}\right)+g\left(\rho_{R}\right)
$$

where:

$$
g(\rho)=\int_{0}^{\rho} \frac{c(a)}{a} d a .
$$

In addition, the solution of the 1D Riemann problem is such that the bound constraints for the void fraction $\alpha$ are fulfilled. We only briefly recall the structure of the solution. The 1 -wave and the 3 - wave admit the following Riemann invariants:

$$
I^{1}=\{u+g(\rho), \alpha\}
$$

and:

$$
I^{3}=\{u-g(\rho), \alpha\}
$$

respectively. Besides, the Riemann invariants in the 2 -wave are:

$$
I^{2}=\{u, P\}
$$

An important point to note is that $\alpha$ may not vary in a 1 -shock or in a 3 -shock. As a consequence, the bound constraints for $\alpha$ cannot be violated. 
Remark. If the condition (14) is violated, a vacuum occurs in the solution, and the uniqueness of the solution $(\rho, u)$ is no longer ensured. Actually, the definition of the velocity in the vacuum aera no longer makes sense in that case (among others, one may refer to [5] for instance which details the construction of the solution $(\rho, \rho u)$ in such a case).

\section{In the remaining of the paper, we will deal with non viscous flows $\mu=0$.}

We now introduce some function $A(x, t)$ such that $A(x, t=0)=x$. We rewrite the governing equations (1)-(3) with $\mu=0$ in the following form:

$$
\begin{aligned}
\frac{\partial A}{\partial t} & =0 \\
\frac{\partial \rho}{\partial t}+\frac{\partial \rho u}{\partial x} & =0 \\
\frac{\partial \rho \alpha}{\partial t}+\frac{\partial \rho \alpha u}{\partial x}-\rho s \frac{\partial A}{\partial x} & =0 \\
\frac{\partial \rho u}{\partial t}+\frac{\partial\left(\rho u^{2}+P(\rho)\right)}{\partial x} & =0
\end{aligned}
$$

The non conservative system (16)-(19) is hyperbolic, since eigenvalues are real and distinct:

$$
\begin{aligned}
& \text { - } \lambda_{1}=0 \\
& \text { - } \lambda_{2}=u \\
& \text { - } \lambda_{3}=u-c \\
& \text { - } \lambda_{4}=u+c
\end{aligned}
$$

unless vacuum arises $(\rho=0)$, or if $u=0$. Fields 3 and 4 are genuinely non linear, and the 1 and 2 fields are linearly degenerated. For convenience, we will use $Z=(A, \alpha, \rho, u)$. Regular solutions of the previous system are governed by:

$$
\begin{aligned}
\frac{\partial A}{\partial t} & =0 \\
\frac{\partial \alpha}{\partial t}+u \frac{\partial \alpha}{\partial x}-s(\alpha) \frac{\partial A}{\partial x} & =0 \\
\frac{\partial \rho}{\partial t}+u \frac{\partial \rho}{\partial x}+\rho \frac{\partial u}{\partial x} & =0 \\
\frac{\partial u}{\partial t}+u \frac{\partial u}{\partial x}+\frac{P^{\prime}(\rho)}{\rho} \frac{\partial \rho}{\partial x} & =0
\end{aligned}
$$

or in a condensed form: $\frac{\partial Z}{\partial t}+C(Z) \frac{\partial Z}{\partial x}=0$. The fields labeled 1 and 2 are linearly degenerated and the Riemann invariants associated with the 1 -wave and the 2 -wave are:

- $J^{1}=\{u, \rho, A+u \phi(\alpha)\}$

- $J^{2}=\{u, \rho, A\}$

where $\phi$ is defined for $s(\alpha) \neq 0$ as:

$$
\phi^{\prime}(\alpha)=-\frac{1}{s(\alpha)}
$$


The fields labeled 3 and 4 are genuinely non linear and the Riemann invariants associated with the 3 -wave and the 4 -wave are:

- $J^{3}=\{u+g(\rho), \alpha, A\}$

- $J^{4}=\{u-g(\rho), \alpha, A\}$

In the limit case where $u=0$, the sets $J^{1}$ and $J^{2}$ coincide.

We now introduce a few notations on figures (1) and (2) depending whether the speed of the contact discontinuity associated with $\lambda_{2}$ is positive or negative respectively. We first detail the

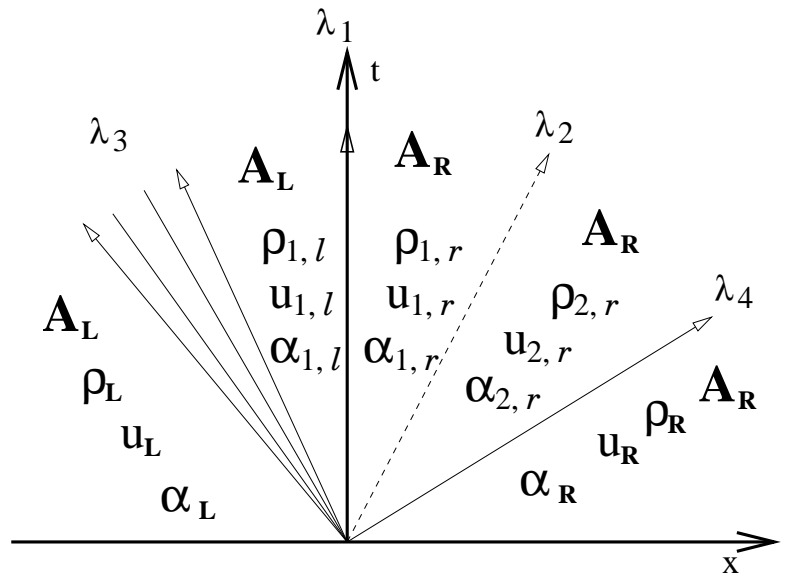

Figure 1: Structure of the solution of the one dimensional Riemann problem $\left(\lambda_{2}>0\right)$

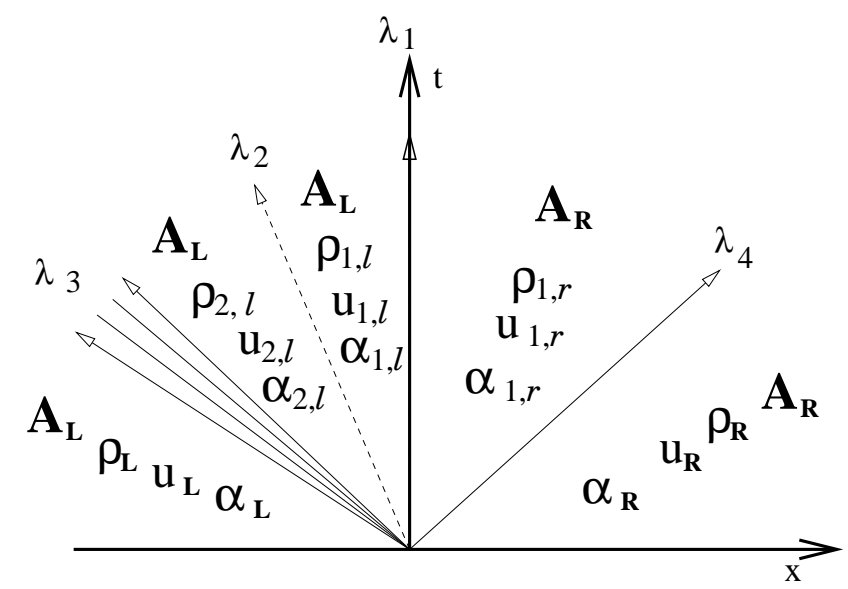

Figure 2: Structure of the solution of the one dimensional Riemann problem $\left(\lambda_{2}<0\right)$

structure of the solution $(\rho, u)$ in the one-dimensional Riemann problem. 
- If $\lambda_{2}>0$, then, owing to $J^{1}$ and $J^{2}$, and due to the fact that both the 1 -wave and the 2 -wave are linearly degenerated, we immediately get $u_{1, l}=u_{1, r}=u_{2, r}=u_{1}$ and $\rho_{1, l}=\rho_{1, r}=\rho_{2, r}=$ $\rho_{1}$.

- If $\lambda_{2}<0$, for the same reasons, we get $u_{2, l}=u_{1, l}=u_{1, r}=u_{1}$ and $\rho_{2, l}=\rho_{1, l}=\rho_{1, r}=\rho_{1}$.

Thus, $u_{1}$ and $\rho_{1}$ will respectively refer to the intermediate velocity and to the intermediate density between the genuinely non linear 3 -wave and the genuinely non linear 4 -wave, and we note $c_{1}=c\left(\rho_{1}\right)$.

Proposition 2a

The one-dimensional Riemann problem associated with (17) and (19) has a unique entropy consistent solution $(\rho, u)$ composed of constant states $\left(\rho_{L}, u_{L}\right),\left(\rho_{1}, u_{1}\right),\left(\rho_{R}, u_{R}\right)$ separated by shock waves or rarefaction waves in the 3 -wave and the 4-wave, with no vacuum occurrence, provided that the initial data $\left(\rho_{L}, u_{L}\right),\left(\rho_{R}, u_{R}\right)$ agrees with the following condition:

$$
u_{R}-u_{L}<g\left(\rho_{L}\right)+g\left(\rho_{R}\right)
$$

$\diamond$

This is a classical result $([12])$.

In order to get some construction of the whole solution, we need to describe more accurately the form of the source term in order to compute the remaining unknown $\alpha$. We will focus below on:

$$
s(\alpha)=\frac{\alpha_{e q}-\alpha}{\tau_{0}}
$$

but the proof that is given is obviously still valid when focusing on many other source terms. If one focuses on regular solutions of equation (21), we first note that it may be classically shown that bound constraints are satisfied (see for instance [11] which recalls this standard result). If one turns to solutions of the Riemann problem, we need the following new result:

Proposition 2b

We assume that the condition (25) holds. Then the bound constraints for the void fraction are guaranteed in the solution of the one dimensional Riemann problem associated with ((16) - (19)), which means that the solution $\alpha(x, t)$ of the Riemann problem lies in $I_{a d m}$.

\section{Proof:}

- Part I:

We will first focus on the case where the intermediate state $u_{1}$ is strictly positive.

In that case, the 3-wave may either behave:

(i) as a subsonic 3-rarefaction wave which spans in $(x<0, t>0)$, when $u_{1}-c_{1}<0$;

(ii) or as a supersonic 3 -rarefaction wave which overlaps $x / t=0$, when $u_{L}-c_{L}<0$ and $u_{1}-c_{1}>0$

(iii) or as a supersonic 3 -rarefaction wave which spans in $(x>0, t>0)$, when $u_{L}-c_{L}>0$; 
(iv) or as a left going 3 -shock wave of speed $\sigma_{3}$;

(v) or as a right going 3 -shock wave of speed $\sigma_{3}$.

In situations (i) and (iv), the velocity along $x / t=0$ is $u_{x=0}=u_{1}$, whereas in case (iii) and (v), it reads $u_{x=0}=u_{L}$. The last possibility is $u_{x=0}=c_{x=0}$ if (ii) occurs. In situations (i), (ii), (iii) and (iv), one thus obtains $u_{x=0}>0$. Making use of Lax inequality, we also check that $u_{L}-c_{L}>\sigma_{3}$ in situation (v), which immediately turns into $u_{x=0}=u_{L}>\sigma_{3}+c_{L}>0$. Hence in all cases, we get $u_{x=0}>0$.

The solution is such that: $\alpha_{1, l}=\alpha_{L}$ and $\alpha_{2, r}=\alpha_{R}$, owing to $J^{3}$ and $J^{4}$, and to the fact that the void fraction $\alpha$ does not vary through $3-4$-shock waves. The remaining unknown is $\alpha_{1, r}$ (see figure (1)):

$$
\begin{array}{cc}
\alpha(x / t)=\alpha_{L} & \text { if } \quad x / t<0 \\
\alpha(x / t)=\alpha_{1, r} \quad \text { if } & 0<x / t<u_{1} \\
\alpha(x / t)=\alpha_{R} & \text { if } \quad x / t>u_{1}
\end{array}
$$

Thus, it only remains to prove that $\alpha_{1, r}$ lies in the admissible range. The solution $\alpha_{1, r}$ fulfills:

$$
A_{R}+u_{x=0} \phi\left(\alpha_{1, r}\right)=A_{L}+u_{x=0} \phi\left(\alpha_{L}\right)
$$

and is thus the solution of the equation:

$$
f(\alpha)=\frac{A_{R}-A_{L}}{u_{x=0}}+\phi(\alpha)-\phi\left(\alpha_{L}\right)=0
$$

where $A_{R}-A_{L}=h$. It remains to check that $\alpha_{1, r}$ belongs to $[0,1]-\left\{\alpha_{e q}\right\}$.

We introduce now $s(\alpha)=\frac{\alpha_{e q}-\alpha}{\tau_{0}}$. We thus get $f^{\prime}(\alpha)=\phi^{\prime}(\alpha)=\frac{-1}{s(\alpha)}=\frac{\tau_{0}}{\alpha-\alpha_{e q}}$. Thus:

- If $\alpha_{L}>\alpha_{e q}$, the unique solution will be :

$$
\alpha_{1, r}=\alpha_{e q}+e^{-\frac{h}{\tau_{0} u_{x=0}}}\left(\alpha_{L}-\alpha_{e q}\right)
$$

By the way we note that $\alpha_{1, r}$ tends towards $\alpha_{e q}$ when $u_{x=0}$ tends towards $0^{+}$, whatever the mesh size is.

- If we turn now to the case where $\alpha_{L}<\alpha_{e q}$, the unique solution of equation (30) is again (31). It may be checked that the solution $\alpha_{1, r}$ lies in $\left(\alpha_{L}, \alpha_{e q}\right)$ in any case.

- Part II:

In a similar way, we may investigate the case $u_{1}<0$.

This leads to the same discussion. Since $\alpha_{2, l}=\alpha_{L}$ and $\alpha_{1, r}=\alpha_{R}$, the remaining unknown is now: $\alpha_{1, l}$ (see figure (2)). This eventually provides the solution

$$
\begin{aligned}
& \alpha(x / t)=\alpha_{L} \quad \text { if } \quad x / t<u_{1} \\
& \alpha(x / t)=\alpha_{1, l} \quad \text { if } \quad u_{1}<x / t<0 \\
& \alpha(x / t)=\alpha_{R} \quad \text { if } \quad x / t>0
\end{aligned}
$$


where $\alpha_{1, l}$ is now :

$$
\alpha_{1, l}=\alpha_{e q}+e^{\frac{h}{\tau_{0} u_{x}=0}}\left(\alpha_{R}-\alpha_{e q}\right)
$$

Note that $u_{x=0}<0$ now. Thus the solution $\alpha_{1, l}$ now lies in $\left(\alpha_{R}, \alpha_{e q}\right)$, thus in $I_{a d m}$. We still check that $\alpha_{1, l}$ tends towards $\alpha_{e q}$ when $u_{x=0}$ tends towards $0^{-}$. 


\section{Standard fractional step method $\left(S_{F S}\right)$}

We want to compute approximations of solutions of the following non-homogeneous system:

$$
\left\{\begin{array}{l}
\frac{\partial W}{\partial t}+\frac{\partial F(W)}{\partial x}=S(W) \\
W(x, 0)=W_{0}(x)
\end{array}\right.
$$

using a fractional step method ([28]). Thus, at each time step, we compute $W^{n+1}$ in terms of $W^{n}$, by solving:

$$
\begin{aligned}
& (P 1): \frac{\partial W^{\circ}}{\partial t}+\frac{\partial F\left(W^{\circ}\right)}{\partial x}=0, W^{\circ}(x, 0)=W^{n}(x) \\
& (P 2): \frac{\partial W}{\partial t}=S(W), W(x, 0)=W^{\circ}(x, \Delta t)
\end{aligned}
$$

successively.

The problem (P1) corresponds to the computation of approximations of solutions of (6)-(8) using the following classical scheme:

$$
\Delta x\left(\left(W_{i}^{\circ}\right)^{n+1}-W_{i}^{n}\right)+\Delta t\left(F\left(W_{i}^{n}, W_{i+1}^{n}\right)-F\left(W_{i-1}^{n}, W_{i}^{n}\right)\right)=0
$$

where $F\left(W_{i}^{n}, W_{i+1}^{n}\right)$ is a numerical flux, which depends on two states $W_{i}^{n}$ and $W_{i+1}^{n}$. This numerical flux will be defined later on (see section 4 where the approximate Godunov scheme based on [4] will be detailed). At this stage, we want to emphasize that some properties of the whole scheme will be tightly linked with the choice of our interface solvers which will provide forms of numerical fluxes.

The problem (P2) requires to look for approximate solutions of the ordinary differential system:

$$
\begin{array}{r}
\frac{\partial \rho \alpha}{\partial t}=\rho s(\alpha) \\
\frac{\partial \rho}{\partial t}=0 \\
\frac{\partial \rho u}{\partial t}=0
\end{array}
$$

At each time step, (37) provides $\rho_{i}^{n+1}=\left(\rho_{i}^{\circ}\right)^{n+1}$ and (38) leads to $u_{i}^{n+1}=\left(u^{\circ}\right)^{n+1}$. Equation (36) is integrated in such a way that the lower and upper bounds of $\alpha^{n+1}$ are ensured. We will use an exact integration of (36) and (37) in our numerical tests.

In the remaining of the paper, this scheme will be refered to as $S_{F S}$. 


\section{Well-balanced schemes and interface Riemann solvers}

\subsection{Interface Riemann solvers}

We still use notations (5), (15) and (24).

Our interface Riemann solvers will require to introduce a change of variable, so that we can work with the new variable $Y(W)$. Moreover, for given left and right states $W_{L}$ and $W_{R}$ in the initial condition, we will define some average $\hat{Y}$ which will depend only on $Y_{L}=Y\left(W_{L}\right)$ and $Y_{R}=Y\left(W_{R}\right)$. Given that average $\hat{Y}$, obvious notations will be used which are: $\hat{\lambda}_{k}=\lambda_{k}(\hat{Y}), \hat{r}_{k}=r_{k}(\hat{Y}), \hat{u}=u(\hat{Y})$, $\hat{\rho}=\rho(\hat{Y}), \hat{c}=c(\hat{Y})$.

In practice, we will focus in this paper on the following classical choice (see [4]):

$$
\hat{Y}_{L R}=\left(Y_{L}+Y_{R}\right) / 2
$$

(other choices such as the one detailed in [7] might be considered).

We also denote for any quantity $\phi:(\Delta \phi)_{L R}=\phi_{R}-\phi_{L}$ and $(\bar{\phi})_{L R}=\left(\phi_{R}+\phi_{L}\right) / 2$. Moreover for any $k=1 . .4, F_{k, l}$ (resp. $F_{k, r}$ ) denotes the value of $F$ on the left (resp. right) side of the Linearly Degenerate field associated with the eigenvalue $\hat{\lambda}_{k}$.

\subsubsection{Basic interface solver}

The left hand side of system (20)-(23) may be rewritten in terms of the non conservative variable $Y(W)={ }^{t}(A, \phi, \rho, u)$ in the form:

$$
\frac{\partial Y}{\partial t}+C(Y) \frac{\partial Y}{\partial x}=0
$$

where:

$$
C(Y)=\left(\begin{array}{cccc}
0 & 0 & 0 & 0 \\
1 & u & 0 & 0 \\
0 & 0 & u & \rho \\
0 & 0 & \frac{c^{2}}{\rho} & u
\end{array}\right)
$$

We turn now to the associated linearized problem:

$$
\frac{\partial Y}{\partial t}+C\left(\hat{Y}_{L R}\right) \frac{\partial Y}{\partial x}=0
$$

By denoting: $\hat{\lambda}_{1}=0, \hat{\lambda}_{2}=\hat{u}, \hat{\lambda}_{3}=\hat{u}-\hat{c}, \hat{\lambda}_{4}=\hat{u}+\hat{c}$, the right eigenvectors $\hat{r}_{k}$ associated with $\hat{\lambda}_{k}$ are respectively:

$$
\begin{array}{r}
\hat{r}_{1}=(\hat{u},-1,0,0) \\
\hat{r}_{2}=(0,1,0,0) \\
\hat{r}_{3}=(0,0, \hat{\rho},-\hat{c}) \\
\hat{r}_{4}=(0,0, \hat{\rho}, \hat{c})
\end{array}
$$


The inverse of the matrix of the right eigenvectors $r_{k}(Y)$ is $(2 \rho c u)^{-1} \omega$, where $\omega$ stands for:

$$
\left(\begin{array}{cccc}
2 \rho c & 0 & 0 & 0 \\
2 \rho c & 2 \rho c u & 0 & 0 \\
0 & 0 & u c & -\rho u \\
0 & 0 & u c & \rho u
\end{array}\right)
$$

The computation of the intermediate states in the linearized solver at each interface between two cells labeled $L, R$ is very easy (see [4] where the basis of the approximate Godunov scheme is recalled). Intermediate states for $(\rho, u)$ components are given through (see figure (3) for notations):

$$
\rho_{1}=\bar{\rho}_{L R}-\frac{\hat{\rho}}{\hat{c}} \frac{(\Delta u)_{L R}}{2} \quad \text { and } \quad u_{1}=\bar{u}_{L R}-\frac{\hat{c}}{\hat{\rho}} \frac{(\Delta \rho)_{L R}}{2}
$$

\subsubsection{A robust interface solver}

We now consider the choice of the non-conservative variable $Y(W)={ }^{t}(A, \phi, g(\rho), u)$ in $(40)$ and (41). The main advantage of the latter is that it enables to preserve positive values for the mean density:

\section{Property:}

No vacuum arises in the intermediate states of this linearized Godunov solver if and only if the initial data does not make vacuum occur in the exact solution of the Riemann problem associated with the non linear set of equations, that is if:

$$
u_{R}-u_{L}<g_{L}+g_{R}
$$

and if the following extra-condition holds:

$$
\int_{0}^{+\infty}\left(\frac{c(a)}{a} d a\right)=+\infty
$$

The first part of the proof is given in [8]. It deeply relies on the fact that the Riemann invariants of the 3-field and the 4-field are linear with respect to $g(\rho)$ and $u$, and that the latter two are components of $Y$. Actually, as proved in [8], this enables to reproduce perfectly rarefaction waves through the approximate linearised solver.

The second extra-condition (47) is also due to the choice of the approximate Godunov scheme, since the latter no longer distinguishes shock waves from rarefaction waves, unlike Godunov scheme of course. The proof may be found in [7].

Before going further on, we emphasize that for standard EOS such as $P(\rho)=P_{0} \rho^{\gamma}$ with $\gamma>1$, we get $g(\rho)=2 c(\rho) /(\gamma-1)$ with $c(\rho)=\left(\gamma P_{0} \rho^{(\gamma-1)}\right)^{1 / 2} ;$ thus, the extra condition (47) is obviously fulfilled.

We now give some details on the discrete values of the void fraction $\alpha$. 


\subsubsection{On the preservation of bound constraints for the void fraction in the interface solvers}

Both "basic and robust" interface solvers provide the same intermediate states of the void fraction which are defined as follows. These require to compute $\phi(\alpha)$ first.

The solution of the linear Riemann problem associated with (41) is classical. One simply needs to compute :

$$
Y_{k, r}-Y_{k, l}=\beta_{k} \hat{r}_{k}
$$

where the $\beta_{k}$ issue from the decomposition of the difference $Y_{R}-Y_{L}$ :

$$
Y_{R}-Y_{L}=\sum_{1}^{4} \beta_{k} \hat{r}_{k}
$$

In order to achieve this, one has to use the forms of the right eigenvectors (42)-(45). Nonetheless, for convenience, one needs to distinguish here whether $\hat{u}_{L R}>0$ or $\hat{u}_{L R}<0$.

- If $\hat{u}_{L R}>0$, left and right states of $\phi$ on each side of the steady contact discontinuity $\frac{x}{t}=$ $\lambda_{1}=0$ are defined below. If $\hat{u}_{L R}-\hat{c}_{L R}$ is positive, we get :

$$
Y_{1, l}-Y_{L}=0
$$

Otherwise, if $\hat{u}_{L R}-\hat{c}_{L R}$ is negative, we get :

$$
Y_{3, r}-Y_{3, l}=\beta_{3} \hat{r}_{3}
$$

which obviously turns to :

$$
Y_{3, r}-Y_{L}=Y_{1, l}-Y_{L}=\beta_{3} \hat{r}_{3}
$$

We also note that the second component of $\hat{r}_{3}$ is null. Hence, we may conclude that in any case:

$$
\phi_{1, l}-\phi_{L}=0
$$

Applying the standard rule (48), we also get:

$$
\phi_{1, r}-\phi_{1, l}=\beta_{1}\left(\hat{r}_{1}\right)_{2}=-\beta_{1}=-\frac{h}{\hat{u}_{L R}}
$$

owing to the form of $\hat{r}_{1}$ detailed in (42).

- If $\hat{u}_{L R}<0$, we may use a similar construction. Thus, we may conclude that the left and right states of $\phi$ on each side of the contact discontinuity $\frac{x}{t}=\lambda_{1}=0$ are:

$$
\phi_{1, l}=\phi_{R}+\frac{h}{\hat{u}_{L R}}
$$

and:

$$
\phi_{1, r}=\phi_{R}
$$


Obviously, $A\left(\frac{x}{t}<0\right)=A_{L}$ and $A\left(\frac{x}{t}>0\right)=A_{R}$. The figure (3) shows the different states for the case $\hat{u}>0$, with $\alpha^{+}=\alpha\left(\phi_{1, r}\right)$ and $\alpha^{-}=\alpha\left(\phi_{1, l}\right)$.

In order to go further on, we are compelled to give the explicit form of the source term, so that we may compute the analytic form of $\phi(\alpha)$ through (24), which eventually will give $\alpha$.

We now restrict to the specific choice:

$$
s(\alpha)=\frac{\alpha_{e q}-\alpha}{\tau_{0}}
$$

Bound constraints for the void fraction in the linearized Riemann problems:

Both previous interface solvers compute intermediate states which are such that the void fraction $\alpha$ remains within its bounds. If $\alpha_{L}$ lies in $\left[0, \alpha_{e q}\left[\right.\right.$ (respectively $\alpha_{L}$ lies in $\left.\left.] \alpha_{e q}, 1\right]\right)$, then for $x / t \in]-\infty, \hat{u}_{L R}$ [, we get: $\alpha_{L} \leq \alpha(x / t)<\alpha_{e q}$ (respectively $\alpha_{e q}<\alpha(x / t) \leq \alpha_{L}$ ). In a similar way, if $\alpha_{R}$ lies in $\left[0, \alpha_{e q}\left[\right.\right.$ (respectively $\alpha_{R}$ lies in $\left.] \alpha_{e q}, 1\right]$ ), then for $\left.x / t \in\right] \hat{u}_{L R},+\infty[$, we get: $\alpha_{R} \leq \alpha(x / t)<\alpha_{e q}$ (respectively $\left.\alpha_{e q}<\alpha(x / t) \leq \alpha_{R}\right)$.

Proof:

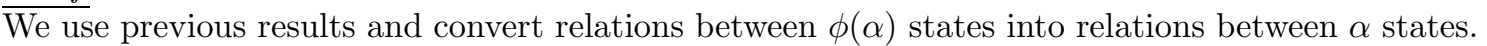

- We first suppose $\hat{u}_{L R}>0$.

Owing to (50), if $x / t \in]-\infty, 0[$, we get:

$$
\alpha(x / t)=\alpha\left(\frac{x}{t}=0^{-}\right)=\alpha_{L}
$$

Otherwise we get, using (51):

$$
\alpha(x / t)=\alpha\left(\frac{x}{t}=0^{+}\right)=\alpha_{e q}+e^{-\frac{h}{\tau_{0} \hat{u}_{L R}}}\left(\alpha_{L}-\alpha_{e q}\right)
$$

if $x / t \in] 0, \hat{u}_{L R}[$.

The last case leads to :

$$
\alpha(x / t)=\alpha_{R}
$$

if $x / t \in] \hat{u}_{L R},+\infty[$.

- On the opposite, if $\hat{u}_{L R}<0$, we get :

$$
\alpha(x / t)=\alpha_{L}
$$

if $x / t \in]-\infty, \hat{u}_{L R}[$.

Otherwise, the counterpart of (52) provides:

$$
\alpha(x / t)=\alpha\left(\frac{x}{t}=0^{-}\right)=\alpha_{e q}+e^{\frac{h}{\tau_{0} \hat{u}_{L R}}}\left(\alpha_{R}-\alpha_{e q}\right)
$$


if $x / t \in] \hat{u}_{L R}, 0[$.

The last possibility is :

$$
\alpha(x / t)=\alpha\left(\frac{x}{t}=0^{+}\right)=\alpha_{R}
$$

if $x / t \in] 0,+\infty[$, which corresponds to (53).

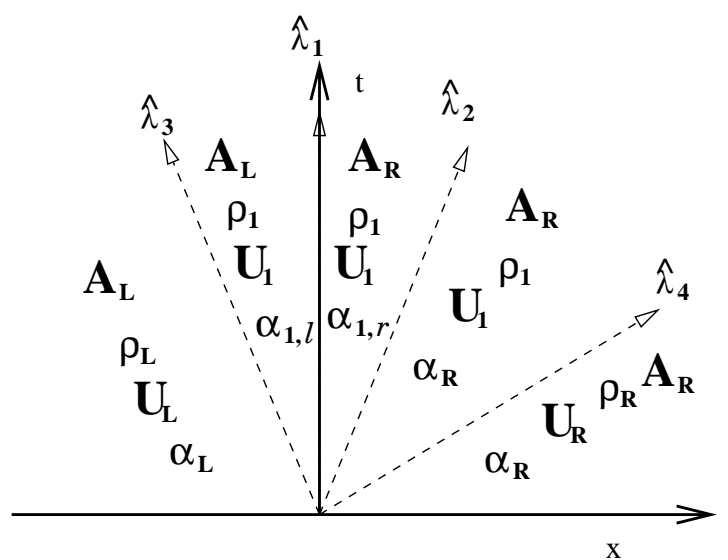

Figure 3: Sketch of the solution of the one-dimensional linearized Riemann problem when $\hat{\lambda}_{2}>0$.

Remark (1). The above property clearly depends on the source term $s(\alpha)$. Bound constraints are also satisfied for a source term of the form $s(\alpha)=\frac{\epsilon}{\tau_{0}} \alpha(1-\alpha)$, which includes two poles, see appendix 5 in [19] for proof.

Remark (2). All computations performed in the next section are based on the use of the first interface Riemann solver with variable $Y(W)={ }^{t}(A, \phi(\alpha), \rho, u)$. It will be combined with the two cell schemes described in the following section.

Remark (3). Using the variable $V(W)={ }^{t}(A, I, g(\rho), u)$ with $I=A+u \phi$ seems to be of interest. Actually the Riemann invariants in the 1-field in the linearized system are exactly conserved. However, $\alpha$ is no longer a Riemann invariant in the 3-field, nor in the 4-field. Thus this change of variables has been disregarded since it does not guarantee that the lower and upper bounds of $\alpha$ will be preserved.

\subsection{Well-balanced schemes}

\subsubsection{First upwinding scheme $S_{U P W 1}$}

For any quantity $D(W(Y))$ chosen among the following: $\rho, \alpha, P, u, \phi, I$, or $\rho u, \rho u \alpha, \rho u^{2}+P(\rho)$, we will use the notation:

$$
D_{i+1 / 2}^{*-}=D\left(W\left(Y^{\text {Riemann }}\left(x / t=0^{-} ; Y_{i}^{n}, Y_{i+1}^{n}\right)\right)\right)
$$


and :

$$
D_{i-1 / 2}^{*+}=D\left(W\left(Y^{\text {Riemann }}\left(x / t=0^{+} ; Y_{i-1}^{n}, Y_{i}^{n}\right)\right)\right)
$$

The solution $Y^{\text {Riemann }}\left(x / t ; Y_{L}, Y_{R}\right)$ stands for the self-similar solution of the Riemann problem associated with (41) and initial condition $Y_{L}$ and $Y_{R}$. The asterisk thus means that we focus on the cell interface $x=0$.

Hence, the approximate Godunov scheme (nicknamed VFRoe-ncv, which has been introduced in [4], and the basics of which have been recalled above), provides:

$$
\begin{aligned}
A_{i}^{n+1}-A_{i}^{n} & =0 \\
\rho_{i}^{n+1}-\rho_{i}^{n}+\frac{\Delta t}{\Delta x}\left((\rho u)_{i+1 / 2}^{*-}-(\rho u)_{i-1 / 2}^{*+}\right) & =0 \\
(\rho \alpha)_{i}^{n+1}-(\rho \alpha)_{i}^{n}+\frac{\Delta t}{\Delta x}\left((\rho \alpha u)_{i+1 / 2}^{*-}-(\rho \alpha u)_{i-1 / 2}^{*+}\right) & =0 \\
(\rho u)_{i}^{n+1}-(\rho u)_{i}^{n}+\frac{\Delta t}{\Delta x}\left(\left(\rho u^{2}+P(\rho)\right)_{i+1 / 2}^{*-}-\left(\rho u^{2}+P(\rho)\right)_{i-1 / 2}^{*+}\right) & =0
\end{aligned}
$$

when applied to our system (16)-(19). The source term $s(\alpha)$ is implicitly contained in the star values, which are given by the interface solver of the previous section. If we take into account (thanks to our interface solver) that $u$ and $\rho$ are continuous at $\frac{x}{t}=0$, and thus that:

$$
u_{i \pm 1 / 2}^{*-}=u_{i \pm 1 / 2}^{*+}=u_{i \pm 1 / 2}^{*}
$$

and:

$$
\rho_{i \pm 1 / 2}^{*-}=\rho_{i \pm 1 / 2}^{*+}=\rho_{i \pm 1 / 2}^{*}
$$

this system can be simplified in:

$$
\begin{aligned}
A_{i}^{n+1}-A_{i}^{n} & =0 \\
\rho_{i}^{n+1}-\rho_{i}^{n}+\frac{\Delta t}{\Delta x}\left((\rho u)_{i+1 / 2}^{*}-(\rho u)_{i-1 / 2}^{*}\right) & =0 \\
(\rho \alpha)_{i}^{n+1}-(\rho \alpha)_{i}^{n}+\frac{\Delta t}{\Delta x}\left((\rho u)_{i+1 / 2}^{*} \alpha_{i+1 / 2}^{*-}-(\rho u)_{i-1 / 2}^{*} \alpha_{i-1 / 2}^{*+}\right) & =0 \\
(\rho u)_{i}^{n+1}-(\rho u)_{i}^{n}+\frac{\Delta t}{\Delta x}\left(\left(\rho u^{2}+P(\rho)\right)_{i+1 / 2}^{*}-\left(\rho u^{2}+P(\rho)\right)_{i-1 / 2}^{*}\right) & =0
\end{aligned}
$$

We can notice that the system $((67),(69))$ is conservative.

In the remaining of the paper, this scheme will be refered to as $S_{U P W 1}$.

\subsubsection{Second upwinding scheme $S_{U P W 2}$}

In section 1 , we have seen that $I=(A+u \phi)$ was continuous in the 1 -wave, we use this particularity to build a second scheme. Equation on the void fraction $\alpha$ is replaced by the non conservative equation using the variable $I$ :

$$
\frac{\partial \rho I}{\partial t}+\frac{\partial \rho u I}{\partial x}+\phi \frac{\partial P}{\partial x}=0
$$


It is easy to verify that this equation is valid for regular solutions. One may also check that jump relations are respected.

The equation (70) is discretized, and thus (62) is replaced by:

$$
(\rho I)_{i}^{n+1}-(\rho I)_{i}^{n}+\frac{\Delta t}{\Delta x}\left((\rho u)_{i+1 / 2}^{*-} I_{i+1 / 2}^{*-}-(\rho u)_{i-1 / 2}^{*+} I_{i-1 / 2}^{*+}\right)+\frac{\Delta t}{\Delta x} \hat{\phi}_{i}^{n}\left(P_{i+1 / 2}^{*-}-P_{i-1 / 2}^{*+}\right)=0
$$

Since we use the same interface Riemann solver, equations (64) and (65) still hold. For all $i$ we note:

$$
\begin{gathered}
I_{i+1 / 2}^{*-}=A_{i}+u_{i+1 / 2}^{*} \phi_{i+1 / 2}^{*-} \\
I_{i+1 / 2}^{*+}=A_{i+1}+u_{i+1 / 2}^{*} \phi_{i+1 / 2}^{*+}
\end{gathered}
$$

where $\phi_{i+1 / 2}^{* \pm}$ is directly computed by the interface solver, using $Y(W)={ }^{t}(A, \phi, \rho, u)$. The equation (71) becomes:

$$
\left.(\rho I)_{i}^{n+1}-(\rho I)_{i}^{n}+\frac{\Delta t}{\Delta x}\left((\rho u)_{i+1 / 2}^{*} I_{i+1 / 2}^{*-}\right)-(\rho u)_{i-1 / 2}^{*} I_{i-1 / 2}^{*+}\right)+\frac{\Delta t}{\Delta x} \hat{\phi}_{i}^{n}\left(P_{i+1 / 2}^{*}-P_{i-1 / 2}^{*}\right)=0
$$

where:

$$
\hat{\phi}_{i}^{n}=\frac{1}{2}\left(\phi_{i+1 / 2}^{*-}+\phi_{i-1 / 2}^{*+}\right)
$$

The next time step requires the computation of $\phi_{i}^{n+1}$ through:

$$
(\rho u)_{i}^{n+1} \phi_{i}^{n+1}=(\rho I)_{i}^{n+1}-\rho_{i}^{n+1} A_{i}
$$

In the remaining of the paper, this scheme will be refered to as $S_{U P W 2}$. 


\section{$5 \quad$ Numerical results}

We now provide some results which have been obtained using the latter three schemes. The three test cases have exact unsteady solutions including discontinuities. In the following, the fractional step method, the scheme with standard upwinding of source terms, and the modified scheme including upwinding of source terms are nicknamed $S_{F S}, S_{U P W 1}$ and $S_{U P W 2}$ respectively.

We will use the following equation of state for our computations:

$$
P(\rho)=K \rho^{2} \quad \text { with: } \quad K=10^{5}
$$

The source term takes the form: $S(\alpha)=\left(\alpha_{e q}-\alpha\right) / \tau_{0}$, with $\alpha_{e q}=0.5$. We have used two distinct values for the time scale which are $\tau_{0}=\left\{10^{-4}, 10^{-6}\right\}$.

\subsection{Expected rates of convergence}

We will use the following notations when computing the $L^{1}$ norm of the error $e(\Delta x, T)$ a time $t=T$ :

$$
e(\Delta x, T)=C(T)(\Delta x)^{\beta}
$$

The (measured) constant $\beta$ will denote the rate of convergence.

Actually, for all test cases, $\alpha$ is governed by :

$$
\frac{\partial \alpha}{\partial t}+u \frac{\partial \alpha}{\partial x}=s(\alpha)
$$

Formally, if the source term is null $s=0$, the expected rate of convergence for $\alpha$ (for a so-called first-order scheme) will be $1 / 2$ (see [9] for instance), since the field $\lambda_{2}=u$ corresponds to a contact discontinuity. Moreover, if $u \frac{\partial \alpha}{\partial x}=0$, the expected rate of convergence for approximate solutions of (78) with help of $S_{F S}$ is 1 . Hence, unless $u \frac{\partial \alpha}{\partial x}=0$ everywhere, $\alpha$ varies through the contact discontinuity associated with $\lambda_{2}=u$, and the leading error will thus be imposed by the convective part on fine meshes (when $\Delta x$ tends towards 0). We may thus expect that the global rate of convergence for $S_{F S}$ will be $\beta_{F S}=1 / 2$.

If we turn to the schemes $S_{U P W 1}$ and $S_{U P W 2}$, the appendix A shows that the source term approximation is also of order 1 with respect to $\Delta t$, and thus of order 1 with respect to $\Delta x$ owing to the CFL constraint:

$$
(|u|+c) \Delta t / \Delta x=C F L_{0}
$$

Therefore, we may also expect that both $S_{U P W 1}$ and $S_{U P W 2}$ asymptotic rates of convergence for approximate solutions of (78) will be equal to the lower order imposed by the convective operator, that is $\beta_{U P W 1}=\beta_{U P W 2}=1 / 2$.

This pattern should be retrieved for fine enough meshes for all schemes. 
According to appendix A, we may also expect that if the non dimensional coefficient $1-e^{-\Delta x /\left(U_{0} \tau_{0}\right)}$ is a poor approximation of $\Delta x /\left(U_{0} \tau_{0}\right)$, which occurs when $\Delta x$ is not small enough, the approximation of the source term connected with $S_{U P W 1}$ will be worse than the one associated with $S_{U P W 2}$ or $S_{F S}$.

\subsection{Description of the numerical tests}

We will focus here on three Riemann problems which involve:

(i) a pure contact discontinuity including a jump of the void fraction $\alpha$;

(ii) a standard shock tube with uniform void fraction over space;

(iii) a standard shock tube with discontinuous initial values of $\alpha$.

We use circles to represent the discrete solution for the first cell scheme $S_{U P W 1}$, while the stars refer to results obtained with the second cell scheme $S_{U P W 2}$. The coarse and the finer mesh contain 100 and 150000 regular cells respectively. For all test cases, the CFL number is set to $C F L_{0}=0.5$. The figure (13) gathers all measured errors for each test case and for the three schemes, when $\tau_{0}=10^{-4}$. The figure (14) corresponds to the measure of the $L^{1}$ norm of the error for the test case (iii) when using scheme $S_{U P W 1}$ and $\tau_{0}=10^{-6}$. All so-called measured rates of convergence have been estimated using the finest two grids.

\subsubsection{Pure contact discontinuity including a jump of the void fraction $\alpha$}

The initial condition of the Riemann problem will be the following:

\begin{tabular}{|l|c|c|}
\hline & LEFT & RIGHT \\
\hline $\mathrm{U}$ & 10 & 10 \\
\hline RHO & 1 & 1 \\
\hline ALPHA & 1 & 0.6 \\
\hline
\end{tabular}

This results in an unsteady contact discontinuity which moves to the right at speed $\sigma=10$. Both the pressure and the velocity remain uniform over time and space: $\rho(x, t)=1$ and $u(x, t)=10$.

In this test case, $u \frac{\partial \alpha}{\partial x}$ is non zero. Actually, the exact solution for the void fraction is:

$$
\left\{\begin{array}{l}
\alpha(x, t)=\alpha_{l}(t), \text { if } x<\sigma t \\
\alpha(x, t)=\alpha_{r}(t), \text { if } x>\sigma t
\end{array}\right.
$$

The function $\alpha_{l}(t)$ (resp. $\left.\alpha_{r}(t)\right)$ is solution of the ODE: $\frac{\partial \alpha}{\partial t}=s(\alpha)$, with initial condition $\alpha(0)=\alpha_{L}$ (resp. with initial condition $\alpha(0)=\alpha_{R}$ ). Of course, both relax to:

$$
\alpha_{e q}=0.5
$$

The time scale is $\tau_{0}=10^{-4}$. In figure (4), the solution is plotted at time Tmax $=3.872910^{-4}$ when the mesh contains 5000 cells. The final values are $\alpha_{l}\left(T_{\max }\right)=0.51039$ and $\alpha_{r}\left(T_{\max }\right)=0.50208$. 
Both the discrete values of velocity and pressure remain constant (thus the error is null). A glance at figure (4) clearly shows that the approximate values of the void fraction which have been computed with schemes $S_{F S}$ and $S_{U P W 2}$ are almost the same. However, though the scheme $S_{U P W 1}$ correctly predicts the position of the contact wave, associated amplitudes of $\alpha$ on both sides are not very accurate on a mesh containing 5000 cells. As shown in appendix A, the local parameter which governs the accuracy on the void fraction, that is: coef $=1-e^{-\Delta x /\left(U_{0} \tau_{0}\right)}$, should be a decent approximation of $\Delta x /\left(U_{0} \tau_{0}\right)$. It thus clearly appears that in the low Mach number regions, the approximation gets worse, and this is obviously depicted in figure (5).

The $L^{1}$ norm of the error for the component $\alpha$ can be found on the top of figure (13). One may note that the measured rate is approximately equal to $\beta_{U P W 1}=0.98, \beta_{U P W 2}=0.55$ and $\beta_{F S}=0.51$ for schemes $S_{U P W 1}, S_{U P W 2}$ and $S_{F S}$ respectively. The rates of convergence for the $\alpha$ component are thus identical for $S_{U P W 2}$ and $S_{F S}$. On each side of the contact discontinuity travelling at speed $\sigma=10$, both discrete values of $u$ and $P$ remain uniform, together with $\alpha$. Thus, following appendix A, schemes $S_{U P W 2}$ and $S_{F S}$ provide the same nice approximation of $\alpha$, which results in a very similar error level on all mesh sizes, and a similar measured rate of convergence which is very close to the asymptotic rate $1 / 2$.

The reason why the measured rate of convergence for $S_{U P W 1}$ is close to 1 , and far from $1 / 2$, is due to the fact that the mesh size of the finest meshes is still too coarse to observe the asymptotic rate of convergence, which is confirmed by the rather high level of the error.

\subsubsection{A standard shock tube with uniform void fraction over space}

Initial condition:

\begin{tabular}{|l|c|c|}
\hline & LEFT & RIGHT \\
\hline $\mathrm{U}$ & 10 & 10 \\
\hline RHO & 1 & 0.5 \\
\hline ALPHA & 1 & 1 \\
\hline
\end{tabular}

The density-velocity solution is composed of a 3 -rarefaction wave and a 4 -shock wave, the 3 -wave expands to the left and the 4 -wave goes to the right boundary. Once again, the void fraction state is relaxed to $\alpha_{e q}$ by the source term, with a one-state pattern (there is no influence of the genuinely non linear fields on the void fraction profile). The time scale is once more $\tau_{0}=10^{-4}$ and the solution is plotted at time $T_{\max }=3.872910^{-4}$ when the mesh contains 5000 cells. The final (uniform) value of the void fraction is $\alpha_{l}\left(T_{\max }\right)=\alpha_{r}\left(T_{\max }\right)=0.51039$. As in the previous case (see figure (5)), the scheme $S_{U P W 1}$ provides a rather poor approximation of the solution $\alpha$, but the error is even more important here, which once more can be easily explained on the basis of Appendix A.

This case is somewhat specific since $u \frac{\partial \alpha}{\partial x}=0$. Thus, the scheme $S_{F S}$ behaves very well, since no error due to the approximation of the convective system arises, which is combined with the fact that $S_{F S}$ exactly integrates source terms within the second step. One should thus keep in mind that this is probably the best set of initial conditions for the latter scheme $S_{F S}$. Actually, the 
error for $S_{F S}$ is almost due to round-off errors, and the measured rate of convergence is meaningless.

On the contrary, the measured rates of convergence for schemes $S_{U P W 1}, S_{U P W 2}$ are approximately equal to $\beta_{U P W 1}=1.005$ and $\beta_{U P W 2}=0.997$ respectively. This means that for that range of meshes, the leading error is still due to the approximation of the source term. As expected, the corrected scheme $S_{U P W 2}$ nonetheless performs much better than $S_{U P W 1}$, as confirmed by the error analysis (see middle of figure (13)). However, the error for $\alpha$ due to $S_{U P W 2}$ is rather small on the left side and on the right side of the computational domain, where $u, \alpha$ and $\rho$ remain uniform -which agrees with the analysis of appendix A -, but is no longer small in the intermediate region where gradients of $u$ and $\rho$ develop. One thus retrieves that the leading error within this range of meshes is still due to the approximation of sources even for the best-suited upwinding scheme $S_{U P W 2}$, which implies that the measured rate of convergence for $S_{U P W 2}$ is still far from the asymptotic rate $1 / 2$.

\subsubsection{A standard shock tube with discontinuous initial values of $\alpha$}

Initial condition:

\begin{tabular}{|l|c|c|}
\hline & LEFT & RIGHT \\
\hline $\mathrm{U}$ & 10 & 10 \\
\hline RHO & 1 & 0.5 \\
\hline ALPHA & 1 & 0.6 \\
\hline
\end{tabular}

The basic solution on $(u, P)$ is the same as in the previous case, but we no longer have the restriction $u \frac{\partial \alpha}{\partial x}=0$. Once again, the void fraction state is "relaxed" to $\alpha_{e q}$ by the source term, with a two-state pattern as in the first case (there is no influence of the genuinely non linear fields on the void fraction profile). The only difference with the first test case is that the speed of the contact discontinuity is now $u=U_{l}=U_{r}$ where the $l, r$ subscripts refer to both sides of the 2-contact discontinuity. Hence, the void fraction discontinuity moves at speed $u \sim 150$. The mesh still contains 5000 cells, the final time is: $T_{\max }=3.872910^{-4}$, and we also have: $\alpha_{l}\left(T_{\max }\right)=0.51039$ and $\alpha_{r}\left(T_{\max }\right)=0.50208$.

Comments pertaining to the three schemes remain almost the same here (see figure (6)). The corrected scheme $S_{U P W 2}$ performs better in the low Mach number regions, unlike the basic upwinding technique $S_{U P W 1}$. However, the approximations provided by $S_{U P W 2}$ are less accurate in the regions where $u$ and $\rho$ profiles develop, compared with zones where both $u$ and $\rho$ are uniform. The scheme $S_{F S}$ still behaves very well.

More precisely, the measured rates of convergence are approximately $\beta_{U P W 1}=0.975, \beta_{U P W 2}=$ 0.842 and $\beta_{F S}=0.501$ for $S_{U P W 1}, S_{U P W 2}$ and $S_{F S}$ respectively (see bottom of figure (13)). For this test case, error levels for the three schemes are much closer to one another, but the patterns mentionned above remain unchanged. The measured rate of convergence for $S_{F S}$ is close to the asymptotic rate $1 / 2$, and the measured rate of convergence for $S_{U P W 1}$ is still close to 1 , though we expect $1 / 2$. 


\subsection{Results on the coarsest mesh $\left(\tau_{0}=10^{-4}\right)$}

We still consider the same test cases but we now only plot results obtained with a coarse mesh with 100 cells (which corresponds to a very coarse mesh such as those used in the industrial framework). We plot separately the results obtained with $S_{F S}$, and $\left(S_{U P W 1}, S_{U P W 2}\right)$.

Pure contact discontinuity including a jump of the void fraction $\alpha$

The initial data has been detailed in section 5.2.1. Results are given in figures (7), (8). The solution is now plotted at time $T \max =3.94010^{-4}$. The final values are now $\alpha_{l}\left(T_{\max }\right)=0.50976$ and $\alpha_{r}\left(T_{\max }\right)=0.50194$

A standard shock tube with uniform void fraction over space

The initial data has been detailed in section 5.2.2. Results are given in figures (9), (10).

A standard shock tube with discontinuous initial values of $\alpha$

The initial data has been detailed in section 5.2.3. Results are given in figures (11), (12).

Remark (4). When $\tau_{0}=10^{-6}$, convergence results for the standard shock tube with discontinuous initial values of $\alpha$ (section 5.2.3) show that (see figure (14)): (i) the error is negligible when using $S_{U P W 2}$ or $S_{F S}$, (ii) the error for $S_{U P W 1}$ (circles) is non negligible on coarse meshes only .

Remark (5). Of course, one should keep in mind the fact that rates of convergence would be better when computing approximations of regular solutions. 


\section{Concluding remarks}

The three schemes converge towards the correct solutions, when focusing on our unsteady tests cases. For all test cases the measured rate of convergence for the fractional step method $S_{F S}$ is close to the asymptotic rate $1 / 2$ (unless meaningless), whereas the measured rate of convergence for the basic upwinding technique $S_{U P W 1}$ is far from the asymptotic rate $1 / 2$ and indeed close to 1 . The measured rate of convergence for the new upwinding approach $S_{U P W 2}$ lies in $[1 / 2,1]$, depending on the test case. For these unsteady test cases, the observed performances indicate that the best approximations are provided by $S_{F S}$, and that those of $S_{U P W 1}$ are rather poor, whatever the mesh size is. We must recall here once more that the basic motivation of $S_{U P W 1}$ concerns the accurate approximation of steady states. The new scheme $S_{U P W 2}$ attempts to benefit from advantages of the upwinding approach (devoted to steady cases) and meanwhile it aims at providing better approximations than $S_{U P W 1}$ in unsteady cases. Results are in agreement with expectations. However, it is not clear whether one may easily derive the counterpart of $S_{U P W 2}$, for any non-homogeneous hyperbolic system.

More precisely, concerning the effective accuracy of schemes, we also have a fourfold conclusion as follows:

- The two shemes $S_{U P W 2}$ and $S_{F S}$ have a similar behavior. The first one $S_{U P W 2}$ is the best one for steady computations, the second one is favoured when highly time dependent solutions develop, especially if convective terms are negligible when compared with (stiff) source terms,

- If $\Delta t \ll \tau_{0}, S_{F S}$ performs quite well,

- The scheme $S_{U P W 1}$ is of course excellent for steady computations,

- If $\frac{\Delta x}{U_{0} \tau_{0}}$ is not small compared with 1 , the scheme $S_{U P W 1}$ provides rather poor numerical results in highly unsteady cases on coarse meshes (though the approximate solution still converges to the exact solution when the mesh size tends to 0). This is obviously not in favour of: (a) low Mach number areas ; (b) small time scales $\tau_{0}$ (that is stiff source terms).

Further improvments are necessary in order to deal with the computation of unsteady solutions of hyperbolic systems with source terms. This is particularly urgent for those who wish to tackle with problems such as those arising in the interfacial coupling of thermohydraulic two-phase flow models (see [1], [2], and [20], [21] for instance). These models involve very different time scales in associated source terms. A straightforward dramatic consequence of the occurence of the stiff source terms is that the accuracy of unsteady computations will diminish when using well-balanced schemes. Actually, it must be noted that the difference of behavior between the fractional step method and the well-balanced scheme is emphasized by this kind of systems. The situation will be different -and the difference between schemes less sensitive- for other non-homogeneous systems such as the shallow water equations with topography, since these involve source terms which directly contribute to the amplitude of the speed of waves (see [10]), but do not contain independent time scales, unless friction terms are accounted for. 
Eventually, one should also note that the use of higher order schemes will not qualitatively change the relative behaviour of both schemes, in a similar framework.

\section{Acknowledgments:}

Authors would like to thank the referee for his very useful comments and criticisms. The second and third authors were supported by the NEPTUNE joint project, which is mainly funded by EDF (Electricité De France), CEA (Commissariat à l'Energie Atomique), with a complementary support by IRSN (Institut de Radioprotection et de Sureté Nucléaire) and AREVA-NP. Computational facilities were provided by Electricité De France. The third author receives financial support by EDF under CIFRE/EDF contract. Part of the work has been achieved while the second author was in LATP, Université Aix-Marseille I, benefiting from financial support by CNRS (Centre National de la Recherche Scientifique). 


\section{References}

[1] A. Ambroso, An introduction to the problem of interfacial coupling of thermohydraulic models, CEA report SFME/LETR/04/008/A, 2004.

[2] A. Ambroso, C. Chalons, F. Coquel, E. Godlewski, F. Lagoutière, P.A. Raviart AND N. SEguin, The coupling of homogeneous models for two-phase flows, submitted for publication.

[3] G.A. BiRD, Molecular gas dynamics and the direct simulation of gas flows, Clarendon, Oxford UK, 1994.

[4] T. Buffard, T. GallouËt and J.M. HÉrard, A sequel to a rough Godunov scheme. Application to real gases, Computers and Fluids, 2000, vol. 29-7, pp. 813-847.

[5] L. Combe, Simulation numérique d'écoulements gaz-particules sur maillage non structuré, PhD thesis, Institut National Polytechnique de Toulouse, Toulouse, France, 1997.

[6] B. Einfeldt, C.D. Munz, P.L. Roe and B. Sjögreen, On Godunov-type methods near low densities, J. Comp. Phys., 1991, vol. 92-2, pp. 273-295.

[7] T. GallouËt, J.M. HÉrard, A new approximate Godunov scheme with application to dense gas-solid flows, AIAA paper 2005-4860, 2005.

[8] T. Gallouët, J.M. Hérard and N. Seguin, On the use of symmetrizing variables for vacuums, Calcolo, 2003, vol. 40(3), pp. 163-194.

[9] T. Gallouët, J.M. Hérard and N. Seguin, Some recent Finite Volume schemes to compute Euler equations using real gas EOS, Int. J. for Num. Meth. in Fluids, 2002, vol. 39(12), pp. 1073-1138.

[10] T. Gallouët, J.M. Hérard and N. Seguin, Some approximate Godunov schemes to compute shallow water equations with topography, Computers and Fluids, 2003, vol. 32(4), pp.479-513.

[11] T. GallouËt, J.M. HÉrard and N. Seguin, Numerical modelling of two-phase flows using the two-fluid two-pressure approach, Mathematical Models and Methods in Applied Sciencces, 2004, vol.14(5), pp.663-700.

[12] E. Godlewski and P.A. Raviart, Numerical approximation of hyperbolic systems of conservation laws, Springer Verlag, 1996.

[13] S.K. Godunov, A difference method for numerical calculation of discontinuous equations of hydrodynamics, Mat. Sb., 1959, pp. 271-300. In Russian.

[14] L. Gosse, A well balanced flux splitting scheme designed for hyperbolic systems of conservation laws with source terms , Computers and Mathematics with Applications, 2000, vol. 39, pp. 135-159.

[15] L. Gosse, A well balanced flux splitting scheme using non conservative products designed for hyperbolic systems of conservation laws with source terms, Math. Mod and Meth. in Appl. Sciences, 2001, vol. 70, pp. 339-365.

[16] L. Gosse And A.Y. LeRoux, Un schéma équilibre adapté aux lois de conservation scalaires non homogènes, Comptes Rendus Académie des Sciences de Paris, 1996, vol. I-323, pp. 543546. 
[17] J.M. Greenberg And A.Y. LeRoux, A well balanced scheme for the numerical processing of source terms in hyperbolic equations, SIAM J. of Num. Anal., 1996, vol. 33, pp. 1-16.

[18] A. Harten, On the symmetric form of systems of conservation laws with entropy, J. Comp. Phys., 1983, vol. 49, pp. 151-164.

[19] J.M. Herard and O. Hurisse, A few schemes to compute hyperbolic systems with source terms, EDF report HI-81/04/07/A, 2004.

[20] J.M. Herard and O. Hurisse, Coupling two and one dimensional unsteady Euler equations through a thin interface, to appear in Computers and Fluids.

[21] J.M. Herard and O. Hurisse, A method to couple HEM and HRM two-phase flow models, EDF report HI-81/06/01/A, submitted for publication.

[22] S. JiN, A steady-state capturing method for hyperbolic systems with geometrical source terms, M2AN, 2001, vol. 35, Num. 4, pp. 631-645.

[23] A.Y. LeRoux, Discrétisation de termes sources raides dans les problèmes hyperboliques, Cours CEA-EDF-INRIA, 1998, INRIA Rocquencourt, France.

[24] A.Y. LeRoux, Oriented characteristics methods for a class of non homogeneous hyperbolic systems, Proceedings of FVCA III, Hermes Penton Science, Raphaele Herbin and Dietmar Kroner editors, 2002, June 24-28, Porquerolles, France.

[25] R.J. LeVeque, Numerical Methods for Conservation Laws, Birkhäuser-Verlag, Basel, 1990.

[26] P.L. RoE, Upwind differencing schemes for hyperbolic conservation laws with source terms, Proceedings of the first International Conference on Hyperbolic Problems. Theory, Numerics and Applications. Carasso, Raviart and Serre editors, 1986.

[27] J. Smoller, Shock waves and reaction diffusion equations, Springer Verlag, 1983.

[28] N.N. Yanenko, Méthode à pas fractionnaires, Armand Colin , 1968. 


\section{Appendix A}

We want here to highlight the relaxation behavior of the different schemes by using particular initial data.

The source term is chosen as :

$$
s(\alpha)=\frac{\alpha_{e q}-\alpha}{\tau_{0}}
$$

We choose the case where $\alpha_{L}=\alpha_{R}$ are greater than $\alpha_{e q}$.

As initial data we choose for all cell $i: u_{i}^{0}=u_{0}>0$ and $P_{i}^{0}=P_{0}$ or equivalently $\rho_{i}^{0}=\rho_{0}$.

The equation (66) gives $A_{i}^{n+1}=A_{i}^{n}$. Due to the VFRoe-ncv scheme, the equation (67) implies that $\rho_{i}^{n+1}=\rho_{i}^{n}$, and the equation (69) implies that $u_{i}^{n+1}=u_{i}^{n}$. Thus we have for all cell $i$ and at any time $t^{n}, u_{i}^{n}=u_{0}$ and $\rho_{i}^{n}=\rho_{0}$.

\section{$7.1 \quad$ Well-balanced scheme $\left(S_{U P W 1}\right)$}

The equation (68) becomes:

$$
\alpha_{i}^{n+1}-\alpha_{i}^{n}+\frac{\Delta t}{\Delta x} u_{0}\left(\alpha_{i+1 / 2}^{*-}-\alpha_{i-1 / 2}^{*+}\right)=0
$$

Using the Riemann solver of section 3.1 and setting $\beta=e^{-\frac{\Delta x}{\tau_{0} u_{0}}}, \lambda=\frac{\Delta t}{\Delta x}$, we have:

$$
\begin{gathered}
\alpha_{i+1 / 2}^{*-}=\alpha_{i}^{n} \\
\alpha_{i-1 / 2}^{*+}=\alpha_{i-1}^{n} \beta+\alpha_{e q}(1-\beta)
\end{gathered}
$$

and equation (62) finally becomes:

$$
\alpha_{i}^{n+1}-\alpha_{i}^{n}+\lambda u_{0}\left(\alpha_{i}^{n}-\alpha_{i-1}^{n}\right)=\lambda u_{0}(1-\beta)\left(\alpha_{e q}-\alpha_{i-1}^{n}\right)
$$

The assumption on the initial values of $\alpha$ leads us to write $\alpha_{i}^{n}=\alpha_{i-1}^{n}=\alpha^{n}$. Thus (79) reads :

$$
\alpha^{n+1}=\alpha^{n}+\lambda u_{0}(1-\beta)\left(\alpha_{e q}-\alpha^{n}\right)
$$

The particular initial data allows us to focus on the relaxation behavior of $\alpha$ due to source term. In order to have a good relaxation behavior, the equation (80) must provide a consistent approximation of the solution of $(36),(37)$, or equivalently of: $\frac{\partial \alpha}{\partial t}=s(\alpha)$, that is:

$$
\alpha\left(x, t^{n}+\Delta t\right)=\alpha\left(x, t^{n}\right) e^{\frac{-\Delta t}{\tau_{0}}}+\alpha_{e q}\left(1-e^{\frac{-\Delta t}{\tau_{0}}}\right)
$$

or at least with the discretized form of $(36),(37)$, which is:

$$
\alpha^{n+1}=\alpha^{n}+\frac{\Delta t}{\tau_{0}}\left(\alpha_{e q}-\alpha^{n}\right)
$$

In order to have a similar behavior the relation $\frac{\Delta t}{\tau_{0}} \sim \lambda u_{0}(1-\beta)$ has to hold. This relation leads to $\frac{\Delta x}{\tau_{0} u_{0}} \sim 0$. Obviously, it will not be satisfied on coarse meshes, especially when either the time scale, or the local velocity is small. 


\subsection{Well-balanced scheme $\left(S_{U P W 2}\right)$}

Inserting the initial data in (71) leads to:

$$
I_{i}^{n+1}-I_{i}^{n}+\lambda u_{0}\left(I_{i}^{n}-I_{i-1}^{n}\right)=0
$$

which can be written:

$$
\phi\left(\alpha_{i}^{n+1}\right)-\phi\left(\alpha_{i}^{n}\right)+\lambda\left(\Delta x+u_{0} \phi\left(\alpha_{i}^{n}\right)-u_{0} \phi\left(\alpha_{i-1}^{n}\right)\right)=0
$$

Thanks to the initial data $\alpha_{i}^{n}=\alpha_{i-1}^{n}=\alpha^{n}$, we get:

$$
\alpha^{n+1}=\left(\alpha^{n}-\alpha_{e q}\right) e^{\frac{-\Delta t}{\tau_{0}}}+\alpha_{e q}
$$

So that to have the same behavior than (82) we must have $\Delta t \ll \tau_{0}$.

\subsection{Fractional step scheme $\left(S_{F S}\right)$}

In the fractionnal step method, $\alpha^{n+1}$ is build up in two steps. First, the Riemann solver computes the convective part of the system ; thus, owing to the specific initial data, the convective step is a ghost step for $\alpha$. The second step is the relaxation due to the source term which is here :

$$
\frac{\partial \alpha}{\partial t}=s(\alpha)=\frac{\alpha_{e q}-\alpha}{\tau_{0}}
$$

Thanks to the form of $s(\alpha)$ we can exactly integrate (86) :

$$
\alpha^{n+1}=\left(\alpha^{n}-\alpha_{e q}\right) e^{\frac{-\Delta t}{\tau_{0}}}+\alpha_{e q}
$$

Note that (87) is the same equation than for the scheme $S_{U P W 2}$. This explains why approximations of $\alpha$ by $S_{U P W 2}$ and $S_{F S}$ are the same in regions where $\alpha, \rho$ and $u$ profiles are uniform.

Hence in order to obtain the same behavior than (82) we must have $\Delta t \ll \tau_{0}$. 


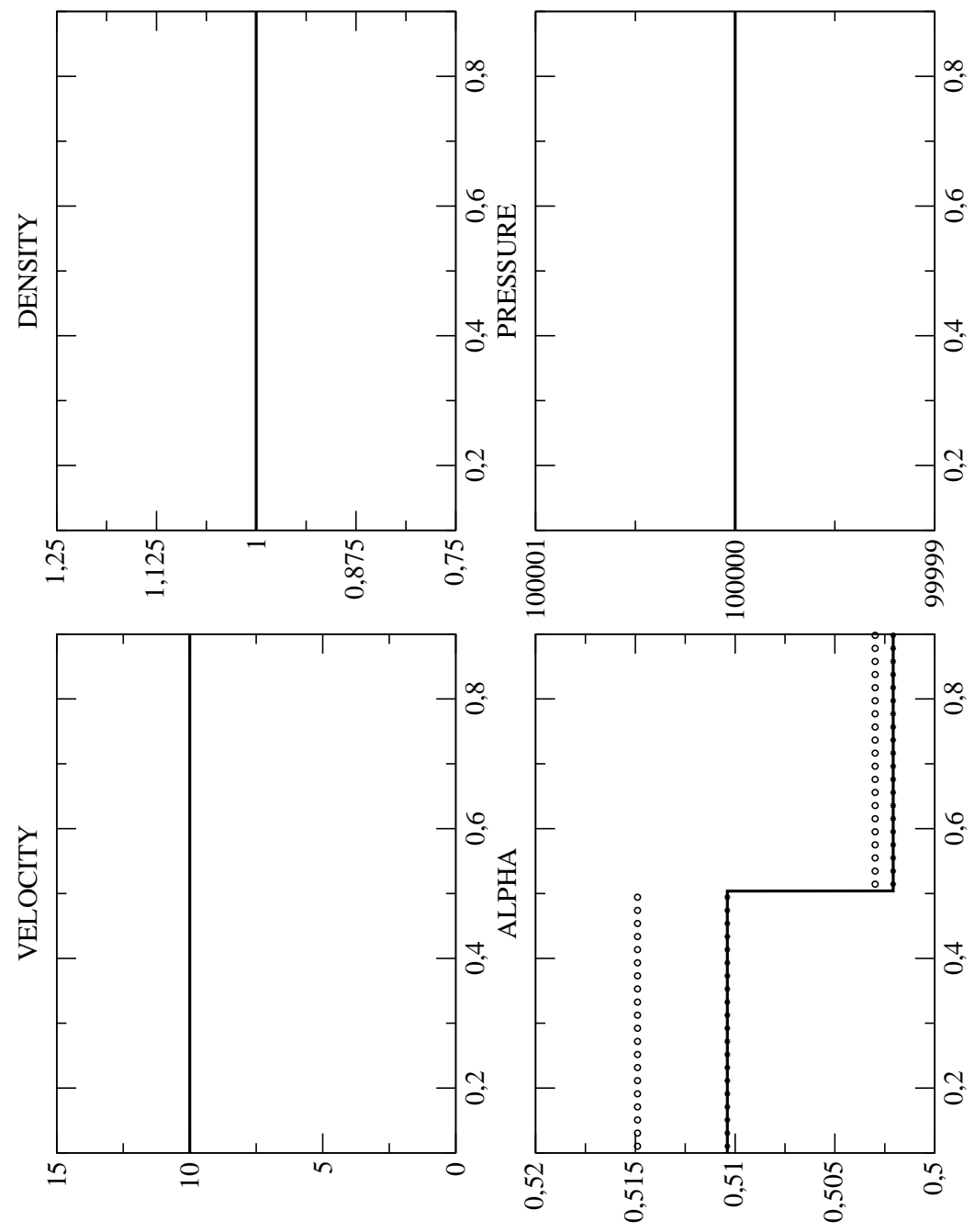

Figure 4: Numerical solutions for the contact wave (5000 cells)

circles $S_{U P W 1}$, black circles $S_{U P W 2}$, continuous line $S_{F S}$.

The exact solution cannot be distinguished from the current approximation obtained with $S_{F S}$. 

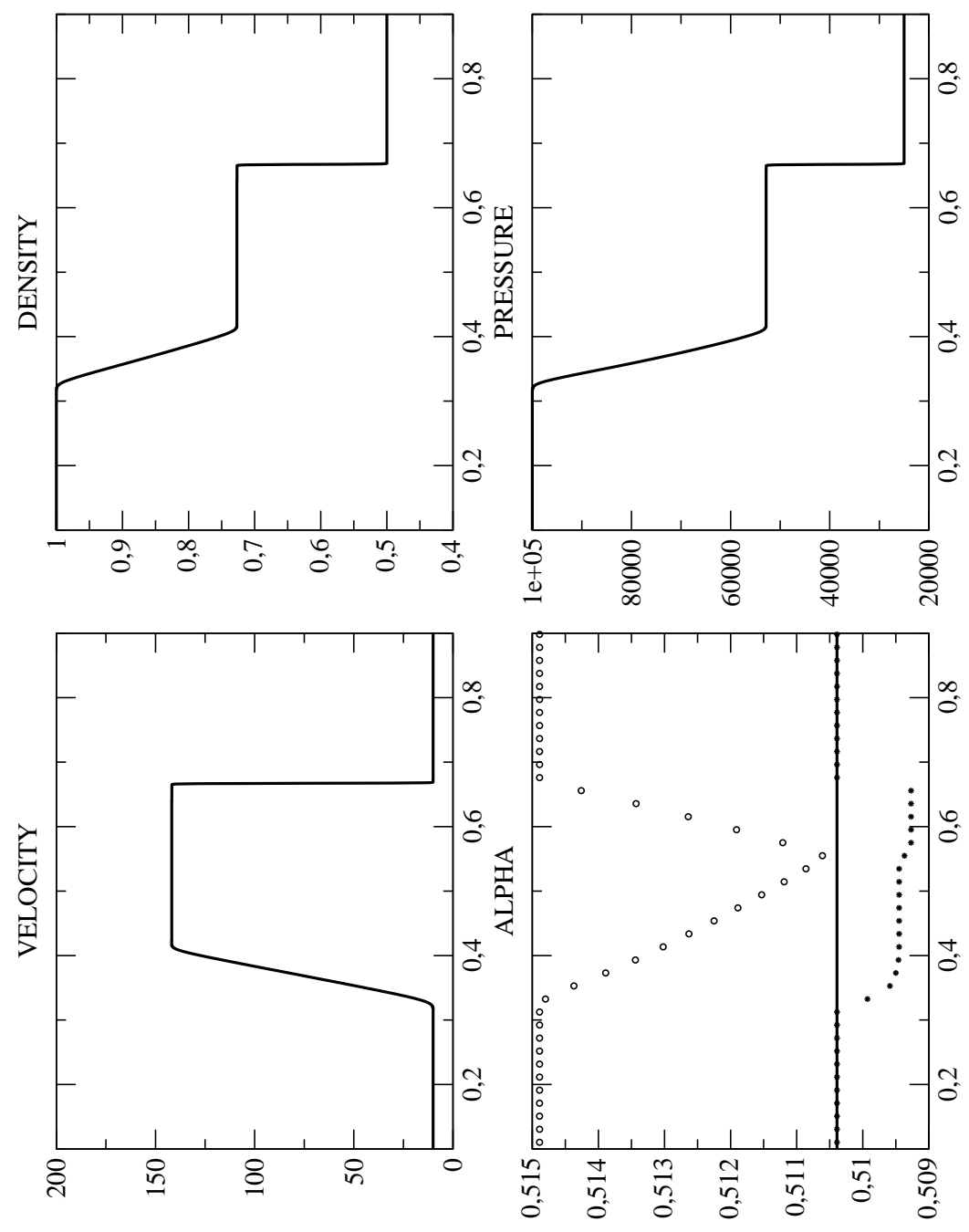

Figure 5: Numerical solutions for the shock tube test case (5000 cells) circles $S_{U P W 1}$, black circles $S_{U P W 2}$, continuous line $S_{F S}$.

The exact solution cannot be distinguished from the current approximation obtained with $S_{F S}$. 

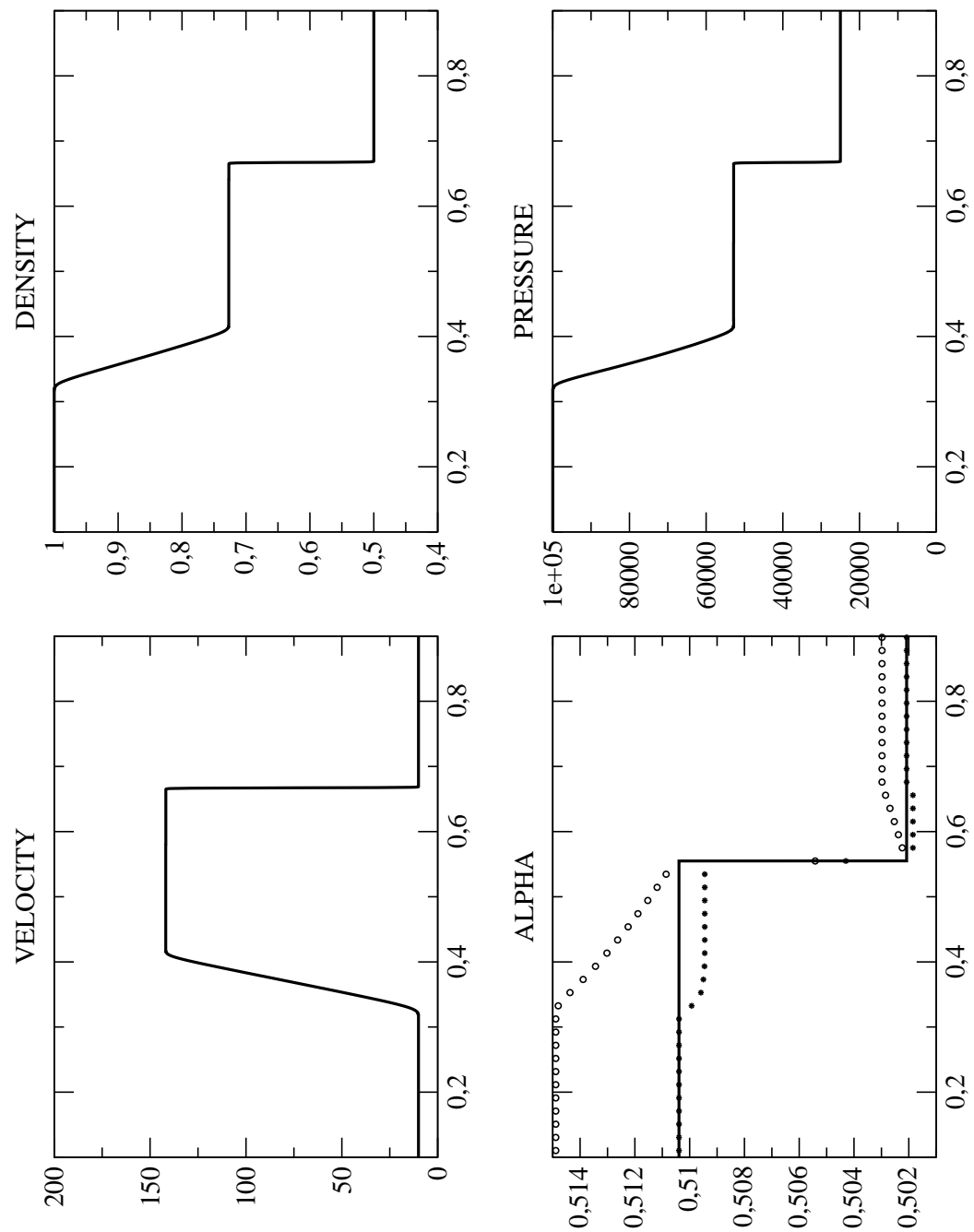

Figure 6: Numerical solutions for the standard shock tube with $\alpha$ discontinuity circles $S_{U P W 1}$, black circles $S_{U P W 2}$, continuous line $S_{F S}$.

The exact solution cannot be distinguished from the current approximation obtained with $S_{F S}$. 


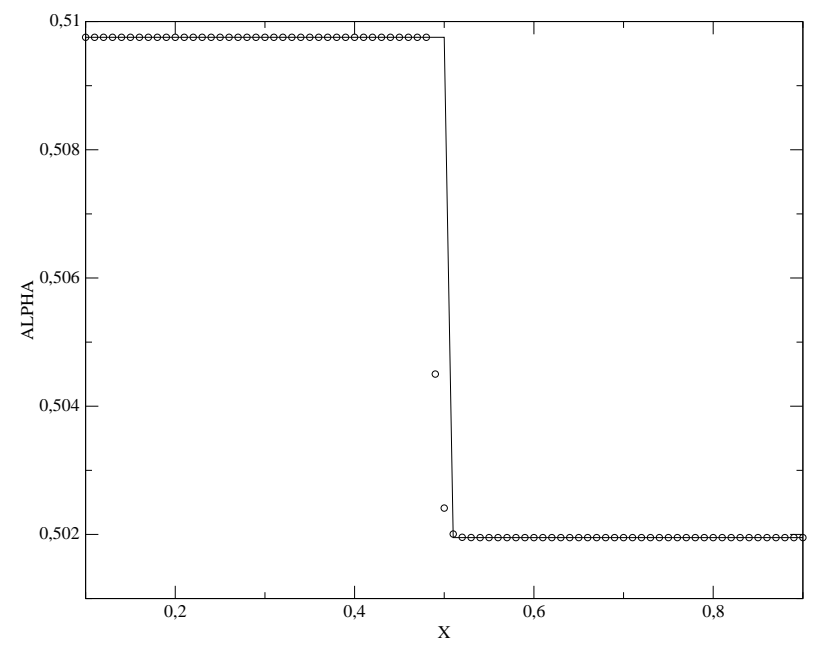

Figure 7: Fractional step method $S_{F S}-100$ cells Pure contact discontinuity.

The continuous line refers to the exact solution.

The initial data is given in section 5.2.1.

$T_{\max }=3.9410^{-4}, \alpha_{l}\left(T_{\max }\right)=0.50976, \alpha_{r}\left(T_{\max }\right)=0.50194$

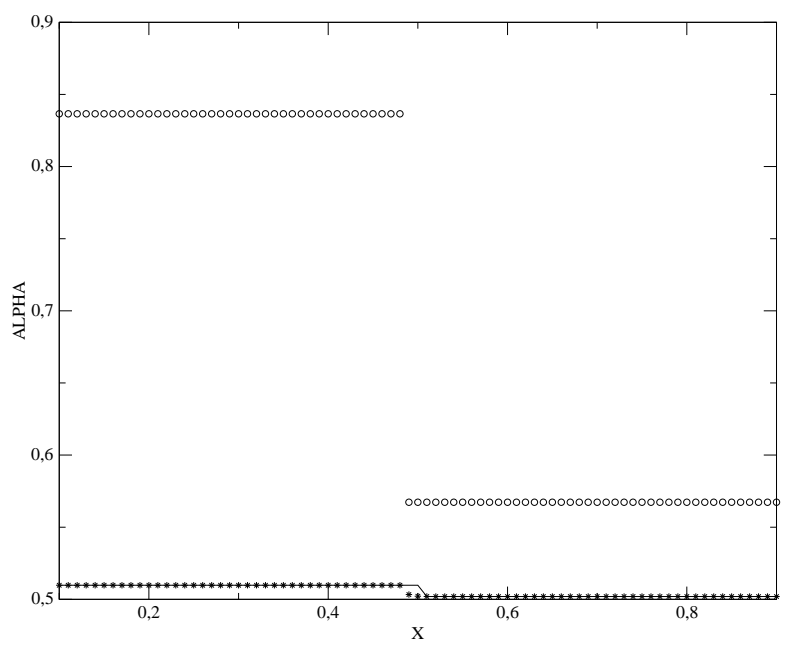

Figure 8: First and second cell schemes for Leroux method $S_{U P W 1}, S_{U P W 2}-100$ cells - The continuous line refers to the exact solution.

The initial data is given in section 5.2.1. 


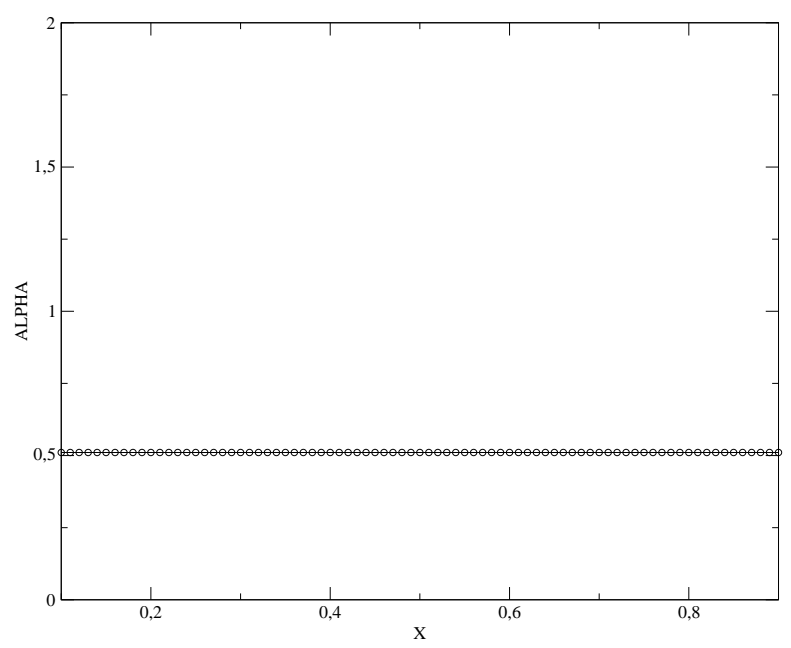

Figure 9: Fractional step method $S_{F S}-100$ cells Shock wave with uniform void fraction profile.

The initial data is given in section 5.2.2.

The continuous line refers to the exact solution.

$T_{\max }=3.872910^{-4}, \alpha_{l}\left(T_{\max }\right)=0.51039, \alpha_{r}\left(T_{\max }\right)=0.51039$

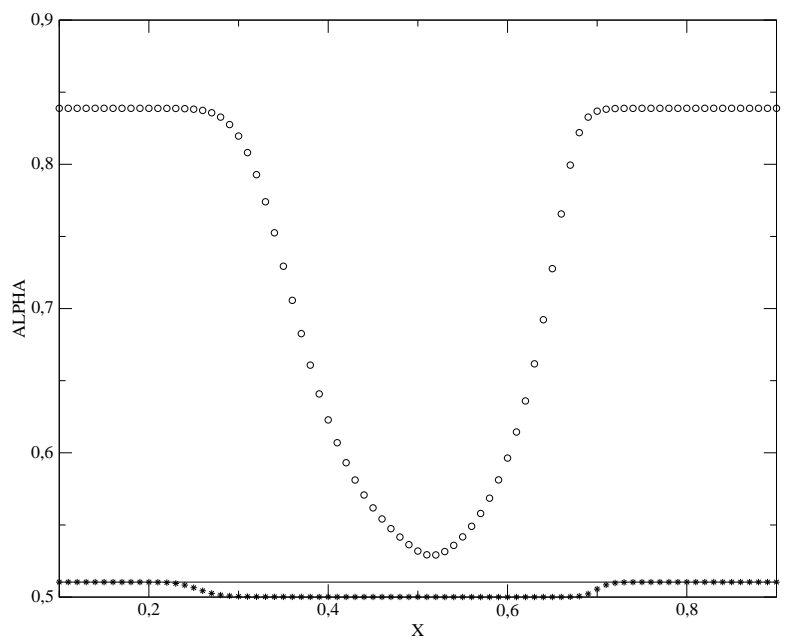

Figure 10: First and second cell schemes for Leroux method $S_{U P W 1}, S_{U P W 2}-100$ cells - The continuous line refers to the exact solution.

The initial data is given in section 5.2.2. 


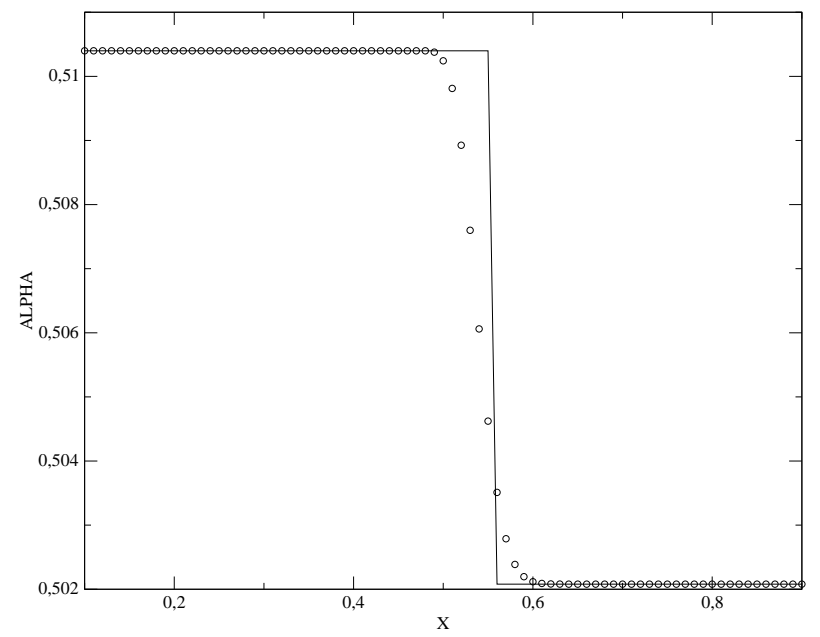

Figure 11: Fractional step method $S_{F S^{-}} 100$ cells -

Shock wave including void fraction desequilibrium.

The initial data is given in section 5.2.3.

The continuous line refers to the exact solution.

$T_{\max }=3.872910^{-4}, \alpha_{l}\left(T_{\max }\right)=0.51039, \alpha_{r}\left(T_{\max }\right)=0.50208$

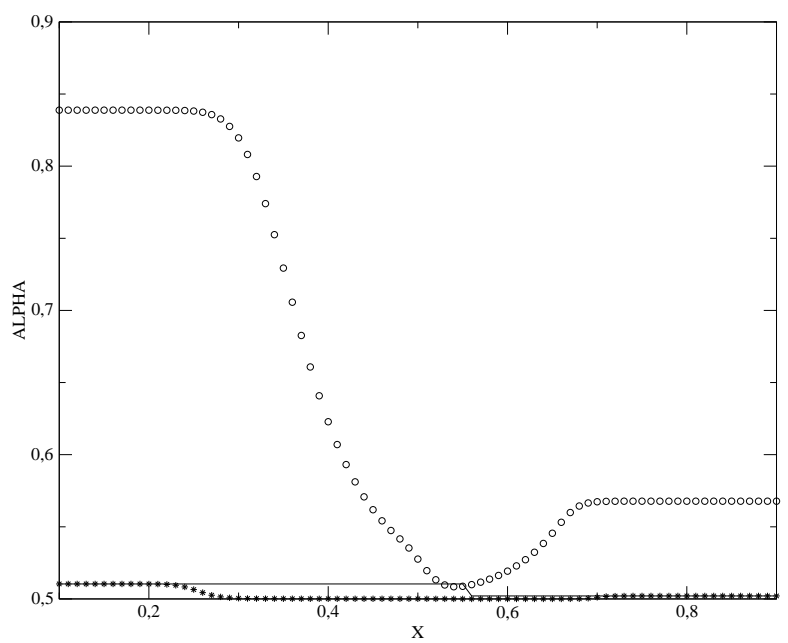

Figure 12: First and second cell schemes for Leroux method $S_{U P W 1}, S_{U P W 2}$ - 100 cells - The continuous line refers to the exact solution. The initial data is given in section 5.2.3. 

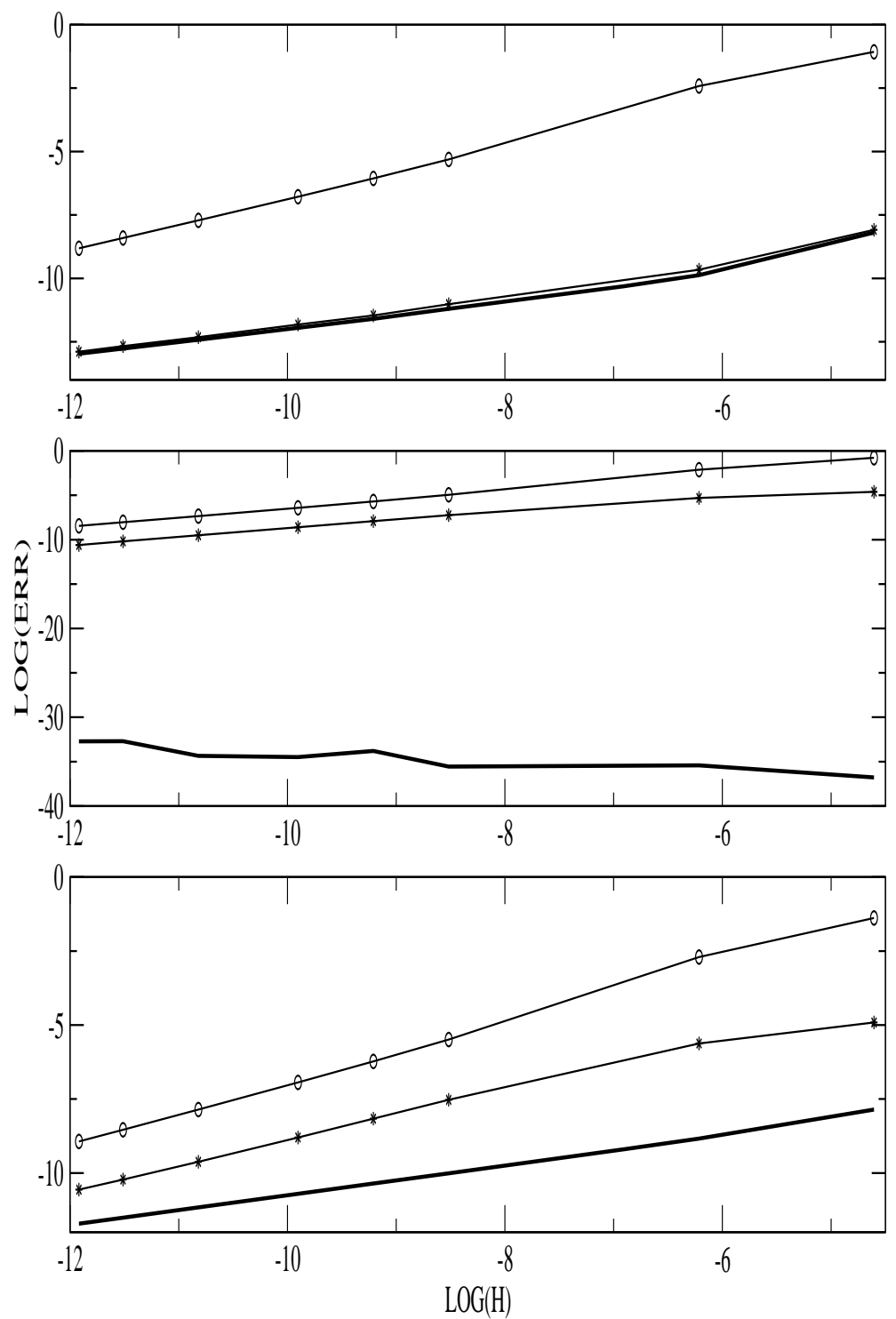

Figure 13: Convergence in $L_{1}$ norm for $\alpha-\tau_{0}=10^{-4}$ -

Pure contact discontinuity, see section 5.2.1 (top)

Shock wave with uniform void fraction profile, see section 5.2.2 (middle)

Shock wave including void fraction desequilibrium, see section 5.2.3 (bottom)

Symbols: circles $S_{U P W 1}$, stars $S_{U P W 2}$, continuous line $S_{F S}$ 


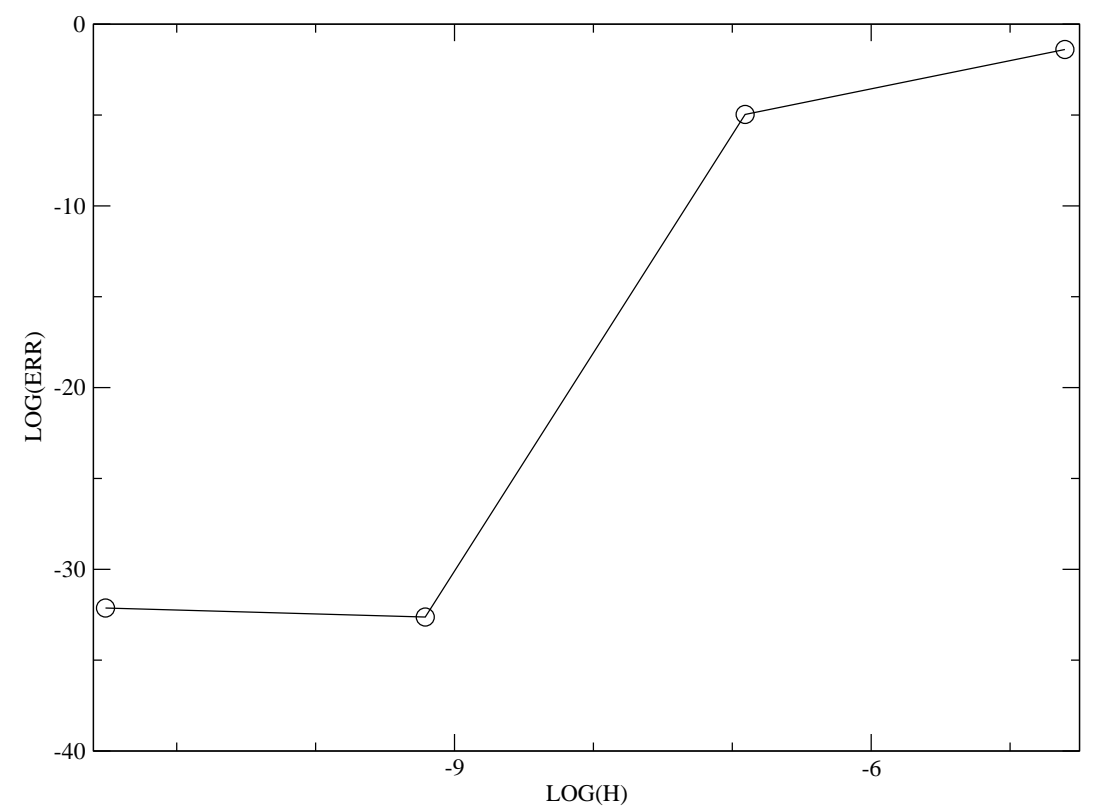

Figure 14: Convergence in $L_{1}$ norm - $\tau_{0}=10^{-6_{-}}$

Shock wave including void fraction desequilibrium circles $S_{U P W 1}$ 